Supporting Information

\title{
Identification of an Active NiCu Catalyst for Nitrile
}

\section{Synthesis from Alcohol}

Yunzhu Wang, ${ }^{\dagger}$ Shinya Furukawa, ${ }^{*, \star,}$ and Ning Yan ${ }^{*, \dagger}$

$\dagger$ Department of Chemical and Biomolecular Engineering, National University of Singapore, 4 Engineering Drive 4, Singapore 117585, Singapore.

$\$$ Institute for Catalysis, Hokkaido University, N-21, W-10, Sapporo 001-0021, Japan.

$\S$ Elements Strategy Initiative for Catalysis and Battery, Kyoto University, Kyoto Daigaku Katsura, Nishikyo-ku, Kyoto, Japan, 615-8510.

*E-mail: furukawa@cat.hokudai.ac.jp.

*E-mail: ning.yan@nus.edu.sg. 


\section{Supplementary experimental procedure}

\section{Materials}

1-Butanol (99\%), 1-hexanol (99\%), 1-heptanol (98\%), 1-octanol (99\%), 1-nonanol (98\%), cyclopentanemethanol (98\%), cyclohexanemethanol (99\%), benzyl alcohol (99\%), 2phenylethanol (99\%), 3-phenyl-1-propanol (98\%), and 4-isopropylbenzyl alcohol (97\%) were purchased from Sigma-Aldrich Pte. Ltd. (Singapore). 1-Butanol-1,1- $\mathrm{d}_{2}(99.5 \%$-d2) was provided by CDN Isotopes (Canada).

\section{Catalyst characterizations}

The crystal structure of the prepared catalyst was examined by powder X-ray diffraction (XRD) with a Rigaku MiniFlex II/AP diffractometer with $\mathrm{Cu} \mathrm{K} \alpha$ radiation. The elemental composition of the catalyst was analyzed by X-ray fluorescence (XRF) spectroscopy using a SHIMADZU Rayny EDX-700HS spectrometer. High angle annular dark field scanning transmission electron microscopy (HAADF-STEM) was carried out using a JEOL JEM-ARM200 M microscope equipped with an energy dispersive X-ray spectroscopy (EDS) analyzer (EX24221M1G5T). The STEM analysis was performed at an accelerating voltage of $200 \mathrm{kV}$. To prepare the TEM specimen, all samples were sonicated in ethanol and then dispersed on a Mo grid supported by an ultrathin carbon film.

The obtained XAS spectra were analyzed using the Athena and Artemis software ver. 0.9.25 included in the Demeter package. ${ }^{1}$ The Fourier transformation of the $k^{2}$-weighted EXAFS from $k$ space to $R$ space was carried out over the $k$ range $3.0-12.5 \AA^{-1}$. A part of the Fourier-transformed EXAFS in the $R$ range of 1.0-3.0 $\AA$ were inversely Fourier-transformed, followed by the analysis with a usual curve fitting method in the $k$ range of $3.0-12.5 \AA^{-1}$. The back-scattering amplitude or phase shift parameters were simulated with FEFF 6L and used to perform the curve 
fitting procedure. The amplitude reduction factors $\left(S_{0}{ }^{2}\right)$ were determined by fitting the spectra of reference samples ( $\mathrm{Ni}$ foil: $0.799, \mathrm{NiO}: 0.833, \mathrm{Cu}$ foil: 0.892 ) and then they were used for fitting of other EXAFS spectra.

\section{Diffuse reflectance infrared Fourier transform spectroscopy (DRIFTS) experiment}

The in-situ DRIFTS spectra were measured on a Thermo Scientific Nicolet iS50 FT-IR under atmospheric pressure, with $\mathrm{ZnS}$ window and a MCT-A detector cooled by liquid nitrogen. Before analysis, catalysts were reduced at $600{ }^{\circ} \mathrm{C}$ under $\mathrm{H}_{2}$ flow for 0.5 hour in the DRIFTS cell. After that, the cell temperature was decreased to $130{ }^{\circ} \mathrm{C}$ under $\mathrm{H}_{2}$ flow. $\mathrm{N}_{2}$ was then purged through the sample cell to remove $\mathrm{H}_{2}$, and the background was recorded under $\mathrm{N}_{2}$. If ammonia was adsorbed before injecting the organic compounds, ammonia was switched on until it was fully saturated on the surface of the catalyst. After that, $\mathrm{N}_{2}$ was turned on to remove the gaseous ammonia as well as physically adsorbed ammonia. Liquid substrate $(10 \mu \mathrm{L})$ was then injected into catalyst cell at the beginning of analysis. The spectra were recorded with $4 \mathrm{~cm}^{-1}$ resolution of 32 scans.

\section{Computational Details for Density functional theory (DFT) calculations}

The DFT method were similar with a previously published article. ${ }^{2}$ The plane-wave basis set was truncated at a kinetic energy of $360 \mathrm{eV}$. A Fermi smearing of $0.1 \mathrm{eV}$ was utilized. Dispersion correlations were considered using the Tkatchenko-Scheffler method with the scaling coefficient of $s_{R}=0.94$ and the damping parameter of $d=20 .{ }^{3}$ The reciprocal space was sampled using a $k$-point mesh with a spacing of typically $0.04 \AA^{-1}$, as generated by the Monkhorst-Pack scheme. ${ }^{4}$ Geometry optimizations and transition state (TS) searches were performed on supercell 
structures using periodic boundary conditions. Solid-solution alloy structure was modeled using a $(2 \times 2 \times 2)$ extended fcc cell having $16 \mathrm{Ni}$ and $16 \mathrm{M}(\mathrm{M}=\mathrm{Fe}, \mathrm{Cu}$ or $\mathrm{Co})$ atoms. For NiZn, the intermetallic body-centered tetragonal $(\mathrm{P} 4 / \mathrm{mmm})$ structure was used. The surfaces were modelled using metallic slabs with a thickness of four atomic layers with $13 \AA$ of vacuum spacing. Unit cell was $(1 \times 1)$ for $\mathrm{NiM}(111)$ surface of the extended fcc cell and $(2 \times 4)$ for $\mathrm{NiZn(101)} \mathrm{surface} \mathrm{(Figure} \mathrm{S13).} \mathrm{The} \mathrm{atomic} \mathrm{arrangement} \mathrm{of} \mathrm{Ni}$ and $\mathrm{M}$ in the bulk and the surface was determined so that a variety of adsorption hollow sites are realized as possible within the limited size of the slab structure. The convergence criteria for structure optimization and energy calculation were set to (a) an SCF tolerance of $1.0 \times 10^{-6} \mathrm{eV}$ per atom, (b) an energy tolerance of $1.0 \times 10^{-5} \mathrm{eV}$ per atom, (c) a maximum force tolerance of $0.05 \mathrm{eV} \AA^{-1}$, and (d) a maximum displacement tolerance of $1.0 \times 10^{-3} \AA$. Spin-polarization was considered for calculation on NiFe.

The adsorption energy was defined as follows: $E_{\mathrm{ad}}=E_{\mathrm{A}-\mathrm{s}}-\left(E_{\mathrm{S}}+E_{\mathrm{A}}\right)$, where $E_{\mathrm{A}-\mathrm{s}}$ is the energy of the slab together with the adsorbate, $E_{\mathrm{A}}$ is the total energy of the free adsorbate, and $E_{\mathrm{S}}$ is the total energy of the bare slab. The adsorption energy for an oxygen-preadsorbed slab was calculated using $E_{\mathrm{SH}}$, the total energy of the oxygen-adsorbed slab, instead of using $E_{\mathrm{S}}$.

The TS search was performed using the complete linear synchronous transit/quadratic synchronous transit (LST/QST) method..$^{5-6}$ Linear synchronous transit maximization was performed followed by energy minimization in the directions conjugating to the reaction pathway. The approximated TS was used to perform QST maximization with conjugate gradient minimization refinements. The cycle was repeated until a stationary point was found. Convergence criteria for the TS calculations were set to root-mean-square forces on an atom tolerance of $0.10 \mathrm{eV} \AA^{-1}$. 


\section{Supplementary Data}

Table S1. Summary of $\mathrm{Ni}_{x} \mathrm{M}_{y} / \mathrm{Al}_{2} \mathrm{O}_{3}$ catalysts used in this work

\begin{tabular}{|c|c|c|c|c|c|c|c|c|}
\hline \multirow{2}{*}{ Catalyst } & \multirow{2}{*}{$x^{a}$} & \multirow{2}{*}{$\mathrm{wt} \%$} & \multirow{2}{*}{$\mathrm{M}^{\mathrm{b}}$} & \multirow{2}{*}{$\mathrm{Y}^{\mathrm{c}}$} & \multicolumn{2}{|c|}{ Temperature $\left({ }^{\circ} \mathrm{C}\right)$} & \multirow{2}{*}{ Phase } & \multirow{2}{*}{ Method } \\
\hline & & & & & calcination & reduction & & \\
\hline \multicolumn{9}{|c|}{ Catalysts in Figure 2(a) } \\
\hline $\mathrm{Ni}_{0.5} \mathrm{Fe}_{0.5}$ & 1 & & $\mathrm{Fe}$ & 1 & 500 & 600 & $\mathrm{Ni}_{0.5} \mathrm{Fe}_{0.5}$ & \multirow{5}{*}{ DP } \\
\hline $\mathrm{Ni}_{0.5} \mathrm{Co}_{0.5}$ & 1 & & Co & 1 & - & 600 & $\mathrm{Ni}_{0.5} \mathrm{Co}_{0.5}$ & \\
\hline $\mathrm{Ni}$ & 1 & 6 & - & - & - & 600 & $\mathrm{Ni}$ & \\
\hline $\mathrm{Ni}_{0.5} \mathrm{Cu}_{0.5}$ & 1 & & $\mathrm{Cu}$ & 1 & 500 & 600 & $\mathrm{Ni}_{0.50} \mathrm{Cu}_{0.50}$ & \\
\hline $\mathrm{Ni}_{0.5} \mathrm{Zn}_{0.5}$ & 1 & & $\mathrm{Zn}$ & 1 & 500 & 600 & $\mathrm{Ni}_{0.5} \mathrm{Zn}_{0.5}$ & \\
\hline \multicolumn{9}{|c|}{ Catalysts in Figure 2(b) } \\
\hline $\mathrm{Ni}_{0.75} \mathrm{Cu}_{0.25}$ & 1 & & $\mathrm{Cu}$ & 1 & 500 & 600 & $\mathrm{Ni}_{0.75} \mathrm{Cu}_{0.25}$ & \multirow{5}{*}{ DP } \\
\hline $\mathrm{Ni}_{0.45} \mathrm{Cu}_{0.55}$ & 1 & 6 & $\mathrm{Cu}$ & 1 & 500 & 600 & $\mathrm{Ni}_{0.45} \mathrm{Cu}_{0.55}$ & \\
\hline $\mathrm{Ni}_{0.26} \mathrm{Cu}_{0.74}$ & 1 & & $\mathrm{Cu}$ & 1 & 500 & 600 & $\mathrm{Ni}_{0.26} \mathrm{Cu}_{0.74}$ & \\
\hline $\mathrm{Ni}_{0.2} \mathrm{Cu}_{0.8}$ & 1 & 20 & $\mathrm{Cu}$ & 1 & 200 & 600 & $\mathrm{Ni}_{0.2} \mathrm{Cu}_{0.8}$ & \\
\hline $\mathrm{Cu}$ & - & - & $\mathrm{Cu}$ & 1 & 500 & 400 & $\mathrm{Cu}$ & \\
\hline \multicolumn{9}{|c|}{ Catalysts in Figure S2(a) } \\
\hline $\mathrm{Ni}_{0.5} \mathrm{Co}_{0.5}$ & 1 & 6 & Co & 1 & - & 600 & $\mathrm{Ni}_{0.5} \mathrm{Co}_{0.5}$ & \multirow{4}{*}{ WI } \\
\hline $\mathrm{Ni}$ & 1 & & - & - & 500 & 600 & $\mathrm{Ni}$ & \\
\hline $\mathrm{Ni}_{0.50} \mathrm{Cu}_{0.50}$ & 1 & & $\mathrm{Cu}$ & 1 & 500 & 600 & $\mathrm{Ni}_{0.50} \mathrm{Cu}_{0.50}$ & \\
\hline $\mathrm{Cu}$ & - & - & $\mathrm{Cu}$ & 1 & 500 & 400 & $\mathrm{Cu}$ & \\
\hline
\end{tabular}
a. $\mathrm{x}=$ molar ratio of $\mathrm{Ni}$
b. $\mathrm{M}=$ second metal
c. $y=$ molar ratio of second metal $\mathrm{M}$

Table S2. Conversion and selectivities of the transformation of 1-butanol to butyronitrile with $\mathrm{Ni} / \mathrm{Al}_{2} \mathrm{O}_{3}, \mathrm{Cu} / \mathrm{Al}_{2} \mathrm{O}_{3}$ and $\mathrm{Ni}_{0.5} \mathrm{Cu}_{0.5} / \mathrm{Al}_{2} \mathrm{O}_{3}$ catalysts prepared with DP method

\begin{tabular}{cccccc}
\hline & Conv. & \multicolumn{4}{c}{ Selectivity (\%) } \\
\cline { 3 - 6 } & (\%) & butyronitrile & 1-butylamine & dibutylamine & others \\
\hline $\mathrm{Ni} / \mathrm{Al}_{2} \mathrm{O}_{3}$ & 13.4 & 85.8 & 6.9 & 0 & 7.3 \\
$\mathrm{Cu} / \mathrm{Al}_{2} \mathrm{O}_{3}$ & 47.9 & 37.2 & 40.3 & 6.7 & 15.8 \\
$\mathrm{Ni}{ }_{0.5} \mathrm{Cu} 0.5 / \mathrm{Al}_{2} \mathrm{O}_{3}$ & 57.2 & 81.5 & 13.6 & 0.1 & 4.8 \\
\hline
\end{tabular}

Reaction conditions: $1 \mu \mathrm{L} / \mathrm{min}$ 1-butanol, $8 \mathrm{~mL} / \mathrm{min} \mathrm{NH}, 20 \mathrm{~mL} / \mathrm{min} \mathrm{N}_{2}, 200 \mathrm{mg}$ catalyst, $160{ }^{\circ} \mathrm{C}, \mathrm{NH}_{3}: 1$-butanol $=30: 1$. 
Table S3. Parameters for the best EXAFS curve fitting with $E_{0}$ calibration $^{\mathrm{a}}$

\begin{tabular}{|c|c|c|c|c|c|c|c|}
\hline sample & shell & $N$ & $R(\AA)$ & $\Delta E(\mathrm{eV})$ & $\sigma^{2}\left(\AA^{2}\right)$ & $R$-factor & $S_{0}^{2}$ \\
\hline $\mathrm{Ni}-\mathrm{K}$ edge & & & & & & & \multirow{6}{*}{$\begin{array}{l}0.799 \\
0.883 \\
1.026 \\
0.883 \\
\text { (fix) } \\
0.799 \\
\text { (fix) }\end{array}$} \\
\hline $\mathrm{Ni}$ foil & $\mathrm{Ni}-\mathrm{Ni}$ & 12 (fix) & 2.484 & 0.00 & 0.006 & 0.000 & \\
\hline $\mathrm{NiO}(F m \overline{3} m)$ & $\mathrm{Ni}-\mathrm{O}$ & 6 (fix) & 2.100 & 0.05 & 0.006 & 0.006 & \\
\hline \multirow{3}{*}{$\mathrm{Ni}_{0.5} \mathrm{Cu}_{0.5} / \mathrm{Al}_{2} \mathrm{O}_{3}$} & $\mathrm{Ni}-\mathrm{Ni}$ & 12 (fix) & 2.950 & -4.02 & 0.007 & \multirow{3}{*}{0.014} & \\
\hline & $\mathrm{Ni}-\mathrm{O}$ & 1.1 & $\begin{array}{l}2.10 \\
\text { (fix) }\end{array}$ & 9.69 & 0.002 & & \\
\hline & $\mathrm{Ni}-\mathrm{Ni}(\mathrm{Cu})$ & 9.1 & 2.513 & 0.04 & 0.008 & & \\
\hline $\begin{array}{l}\text { Cu-K edge } \\
\mathrm{Cu} \text { foil }\end{array}$ & $\mathrm{Cu}-\mathrm{Cu}$ & 12 (fix) & 2.542 & -0.02 & 0.009 & 0.001 & \multirow{3}{*}{$\begin{array}{l}0.892 \\
0.892 \\
\text { (fix) } \\
0.892 \\
\text { (fix) }\end{array}$} \\
\hline $\mathrm{Cu} / \mathrm{Al}_{2} \mathrm{O}_{3}$ & $\mathrm{Cu}-\mathrm{Cu}$ & 8.0 & 2.540 & 0.03 & 0.009 & 0.010 & \\
\hline $\mathrm{Ni}_{0.5} \mathrm{Cu}_{0.5} / \mathrm{Al}_{2} \mathrm{O}_{3}$ & $\mathrm{Cu}-\mathrm{Cu}(\mathrm{Ni})$ & 8.8 & 2.509 & -0.01 & 0.010 & 0.002 & \\
\hline
\end{tabular}

a. For precise comparison, each initial $E_{0}$ was calibrated so that $\Delta E$ of the main shell was less than 0.05 . $k$ window: $3 \sim 12.5$.

Table S4. Estimated particle size according to idealized hexagonal close-packed full-shell metal clusters.

\begin{tabular}{ccccccc}
\hline $\begin{array}{c}\text { Number } \\
\text { of } \\
\text { shells }\end{array}$ & $\begin{array}{c}\text { Total } \\
\text { number of } \\
\text { atoms }\end{array}$ & $\begin{array}{c}\text { Number of } \\
\text { surface } \\
\text { atoms }\end{array}$ & $\begin{array}{c}\text { Surface } \\
\text { atom } \\
\text { percentage } \\
(\%)\end{array}$ & $\begin{array}{c}\text { Estimated } \\
\text { particle size } \\
\text { of Ni (nm) }\end{array}$ & $\begin{array}{c}\text { Estimated } \\
\text { particle size } \\
\text { of Cu (nm) })^{\mathrm{a}}\end{array}$ & $\begin{array}{c}\text { Estimated } \\
\text { particle size } \\
\text { of NiCu } \\
(\mathrm{nm})^{\mathrm{a}}\end{array}$ \\
\hline 1 & 13 & 12 & 92.3 & 0.74 & 0.76 & 0.75 \\
2 & 55 & 42 & 76.4 & 1.24 & 1.27 & 1.26 \\
3 & 147 & 92 & 63.6 & 1.74 & 1.78 & 1.76 \\
4 & 309 & 162 & 52.4 & 2.23 & 2.29 & 2.26 \\
5 & 561 & 252 & 44.9 & 2.73 & 2.80 & 2.76 \\
15 & 12431 & 2252 & 18.1 & 7.70 & 7.88 & 7.79 \\
16 & 14993 & 2562 & 17.1 & 8.19 & 8.39 & 8.29 \\
\hline
\end{tabular}

a. The $\mathrm{M}-\mathrm{M}$ bond lengths of $\mathrm{NiCu}$ (intermediate value, $2.513 \AA$ ), $\mathrm{Ni}(2.483 \AA$ ), and $\mathrm{Cu}(2.542$ Å) were extracted from Table S3. 
Table S5. Relative metal content before and after catalytic reaction $\left(210^{\circ} \mathrm{C}, 4 \mathrm{~h}\right)$ determined by XRF

\begin{tabular}{ccc}
\hline \multirow{2}{*}{ Catalyst } & \multicolumn{2}{c}{ Relative ratio } \\
\cline { 2 - 3 } & $\mathrm{Ni}$ & $\mathrm{Cu}$ \\
\hline $\mathrm{Ni}_{0.5} \mathrm{Cu}_{0.5} / \mathrm{Al}_{2} \mathrm{O}_{3}$ & & \\
fresh & 1.00 & 1.00 \\
spent & 1.02 & 0.99 \\
\hline $\mathrm{Cu} / \mathrm{Al}_{2} \mathrm{O}_{3}$ & & \\
fresh & - & 1.00 \\
spent & - & 0.98 \\
\hline
\end{tabular}

Table S6. Percentage of compounds which cannot be desorbed after stabilization ${ }^{\mathrm{a}}$

\begin{tabular}{cccc}
\hline & $\mathrm{NiCu}$ & $\mathrm{Ni}$ & $\mathrm{Cu}$ \\
\hline 1-Butanol & $0 \%$ & $1.5 \%$ & $0.5 \%$ \\
Butanal & $0 \%$ & $0 \%$ & $7.4 \%$ \\
1-Butylamine & $82 \%$ & $55 \%$ & $62 \%$ \\
Butyronitrile & $0.8 \%$ & $2.1 \%$ & $2.3 \%$ \\
\hline
\end{tabular}

a. At $\mathrm{t}=0,10 \mu \mathrm{l}$ of organic compounds were injected under $\mathrm{N}_{2}$ flow, $130^{\circ} \mathrm{C}$.

The final spectra were collected after stabilization for more than 30 mins. Near quantitative amount of 1-butanol, butanal and butyronitrile were desorbed from the metal surface, whereas $82 \%$ 1-butylamine remained on $\mathrm{NiCu}$ surface after stabilization, more than on $\mathrm{Ni}$ and $\mathrm{Cu}$ surface. The results suggested that the adsorbed 1-butylamine could be further converted to butyronitrile on $\mathrm{NiCu}$ surface, which promoted butyronitrile production. 
Table S7. $E_{\mathrm{ad}}, E_{\mathrm{a}}$, and $\Delta E$ in $\mathrm{C}-\mathrm{H}$ activation of methoxide to formaldehyde on $\mathrm{Ni}$ and $\mathrm{NiM}$ surfaces.

\begin{tabular}{ccccc}
\hline \multirow{2}{*}{ Entry } & \multirow{2}{*}{ Configuration } & \multicolumn{3}{c}{ Energy $(\mathrm{kJ} / \mathrm{mol})$} \\
\cline { 3 - 4 } & & $E_{\mathrm{ad}}(\mathrm{OMe})$ & $\Delta E(\mathrm{C}-\mathrm{H})$ & $E_{\mathrm{a}}(\mathrm{C}-\mathrm{H})$ \\
\hline 2 & $\mathrm{Ni}$ & -246.7 & 12.2 & 83.3 \\
3 & $\mathrm{NiCu}-2$ & -216.5 & 9.0 & 74.0 \\
4 & $\mathrm{NiCo}-2$ & -232.3 & 33.9 & 105.4 \\
5 & $\mathrm{NiFe}-2$ & -242.1 & 43.5 & 105.8 \\
6 & $\mathrm{NiZn}$ & -195.8 & 61.0 & 131.4 \\
7 & $\mathrm{Ni}(2 \mathrm{HB})$ & -206.4 & -14.2 & 63.4 \\
8 & $\mathrm{NiCu}-2(2 \mathrm{HB})$ & -213.9 & -27.3 & 49.9 \\
9 & $\mathrm{NiCo}-2(2 \mathrm{HB})$ & -199.6 & -28.7 & 67.4 \\
10 & $\mathrm{NiFe}-2(2 \mathrm{HB})$ & -220.4 & -0.1 & 82.9 \\
& $\mathrm{NiZn}(2 \mathrm{HB})$ & -174.2 & 20.1 & 99.3 \\
\hline
\end{tabular}



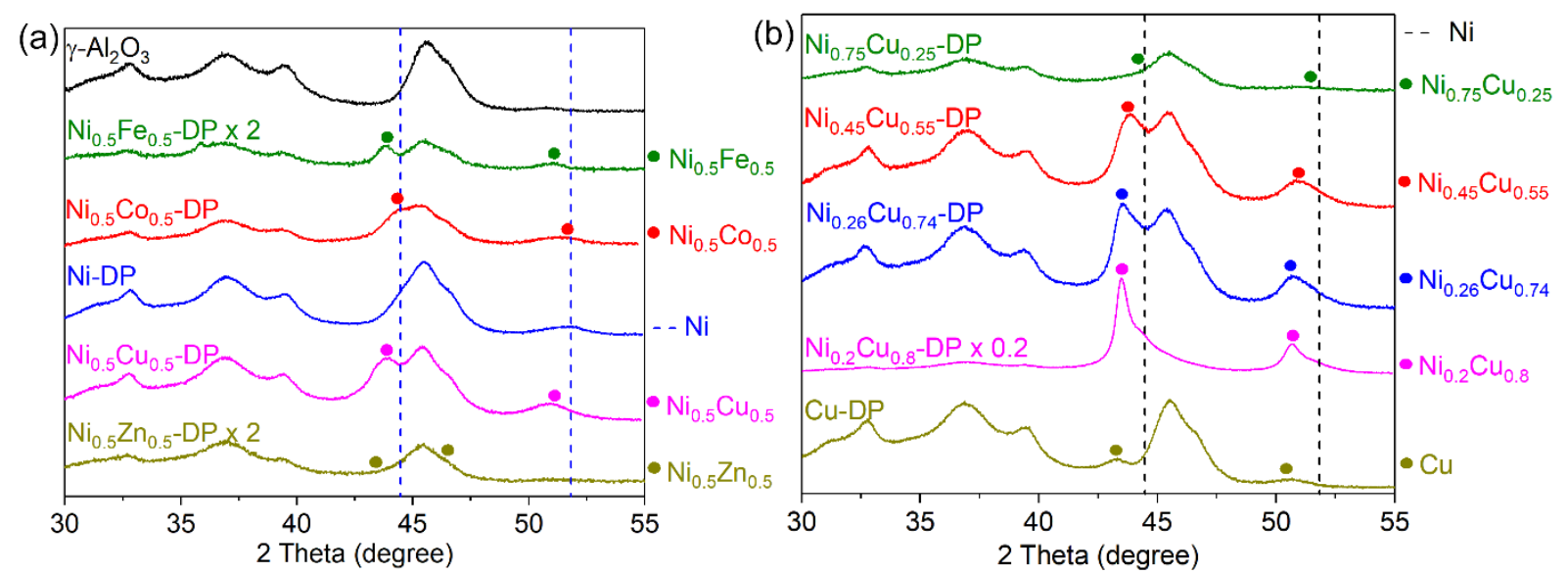

Figure S1. XRD patterns of (a) various $\mathrm{NiM} / \mathrm{Al}_{2} \mathrm{O}_{3}$ and $\mathrm{Ni} / \mathrm{Al}_{2} \mathrm{O}_{3}$ catalysts prepared with $\mathrm{DP}$ method, (b) various $\mathrm{NiCu} / \mathrm{Al}_{2} \mathrm{O}_{3}$ and $\mathrm{Cu} / \mathrm{Al}_{2} \mathrm{O}_{3}$ catalysts prepared with DP method.

The peak intensity of $\mathrm{Ni}_{0.5} \mathrm{Fe}_{0.5} / \mathrm{Al}_{2} \mathrm{O}_{3}$ (2 times), $\mathrm{Ni}_{0.5} \mathrm{Zn}_{0.5} / \mathrm{Al}_{2} \mathrm{O}_{3}$ (2 times) and $\mathrm{Ni}_{0.2} \mathrm{Cu}_{0.8} / \mathrm{Al}_{2} \mathrm{O}_{3}$ ( 0.2 times) were modified to clearly show the position of alloy peak.
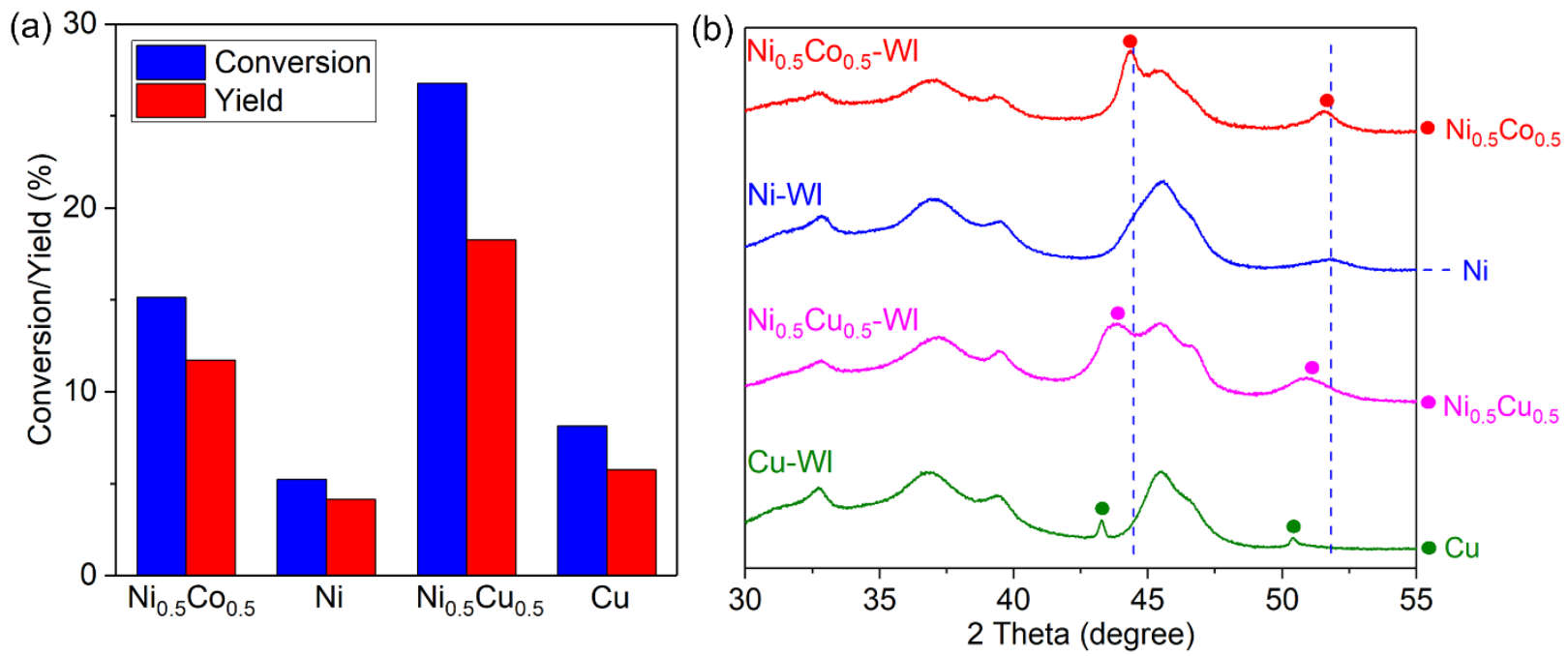

Figure S2. (a) Catalytic activities of converting 1-butanol to butyronitrile with various $\mathrm{NiM} / \mathrm{Al}_{2} \mathrm{O}_{3}$ catalysts prepared with WI method. Reaction conditions: $1 \mu \mathrm{L} / \mathrm{min}$ 1-butanol, 8 $\mathrm{mL} / \mathrm{min} \mathrm{NH}_{3}, 20 \mathrm{~mL} / \mathrm{min} \mathrm{N}$, $200 \mathrm{mg}$ catalyst (or $60 \mathrm{mg} \mathrm{Ni} 0_{2} \mathrm{Cu}_{0.8}$ ), $160{ }^{\circ} \mathrm{C}, \mathrm{NH}_{3}: 1$-butanol $=$ 30:1. (b) XRD patterns of catalysts prepared by WI methods. 

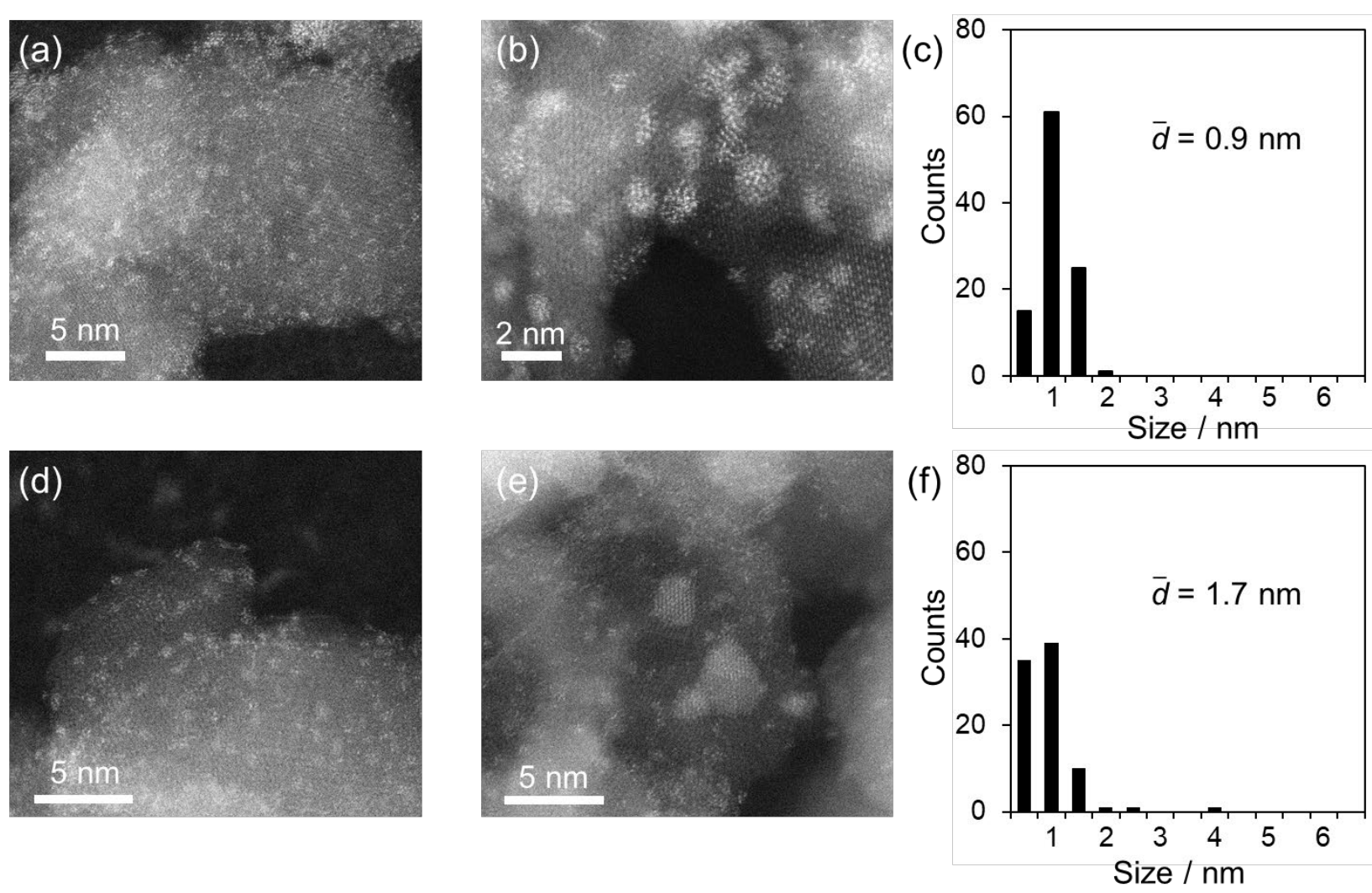

Figure S3. HAADF-STEM images and size distribution of (a-c) $\mathrm{Ni} / \mathrm{Al}_{2} \mathrm{O}_{3}$ and (d-e) $\mathrm{Cu} / \mathrm{Al}_{2} \mathrm{O}_{3}$.
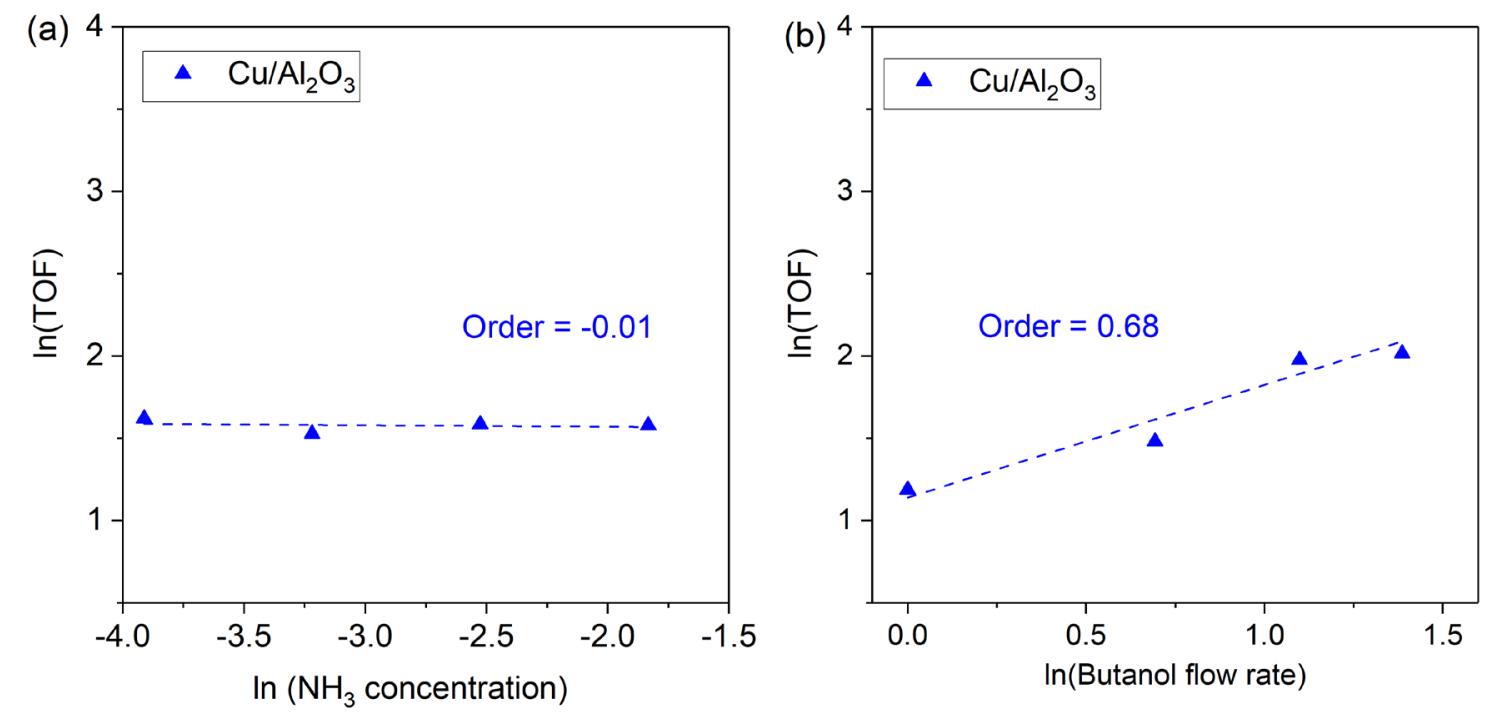

Figure S4. Plots of reaction orders for the conversion of 1-butanol to butylnitrile, of (a) $\mathrm{NH}_{3}$, reaction conditions: $3 \mathrm{ul} / \mathrm{min}$ 1-butanol with $80 \mathrm{mg} \mathrm{Cu} / \mathrm{Al}_{2} \mathrm{O}_{3}, 100 \mathrm{ml} / \mathrm{min}$ total gas flow rate with supplementing $\mathrm{N}_{2}, 210{ }^{\circ} \mathrm{C}$; (b) 1-butanol, reaction conditions: 1 to $4 \mathrm{ul} / \mathrm{min} 1$-butanol, 8 $\mathrm{ml} / \mathrm{min} \mathrm{NH} \mathrm{H}_{3}, 92 \mathrm{ml} / \mathrm{min} \mathrm{N}_{2}, 100 \mathrm{mg} \mathrm{Cu} / \mathrm{Al}_{2} \mathrm{O}_{3}, 210^{\circ} \mathrm{C}$. 


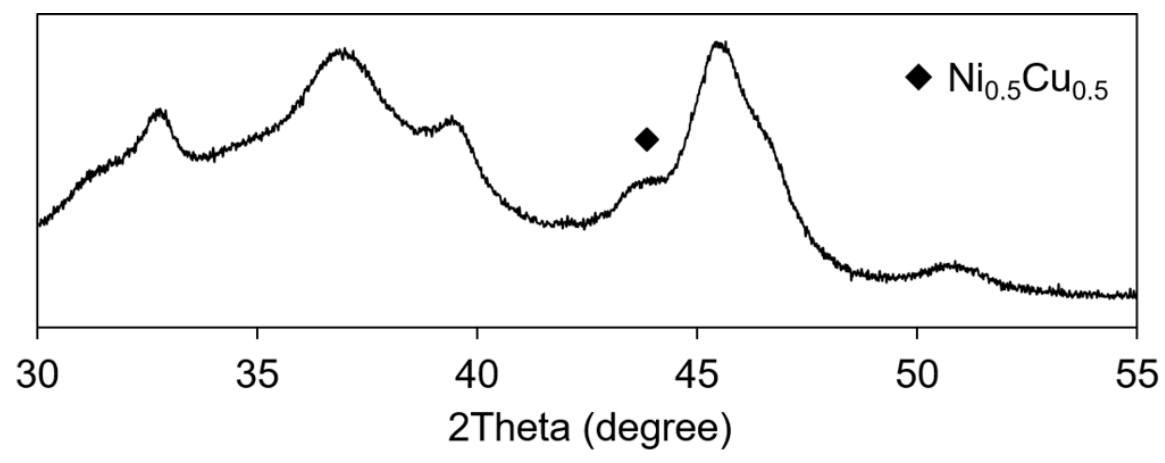

Figure S5. XRD pattern of $\mathrm{Ni}_{0.5} \mathrm{Cu}_{0.5} / \mathrm{Al}_{2} \mathrm{O}_{3}$ catalyst after catalytic reaction $\left(210{ }^{\circ} \mathrm{C}, 4 \mathrm{~h}\right)$.

(a)

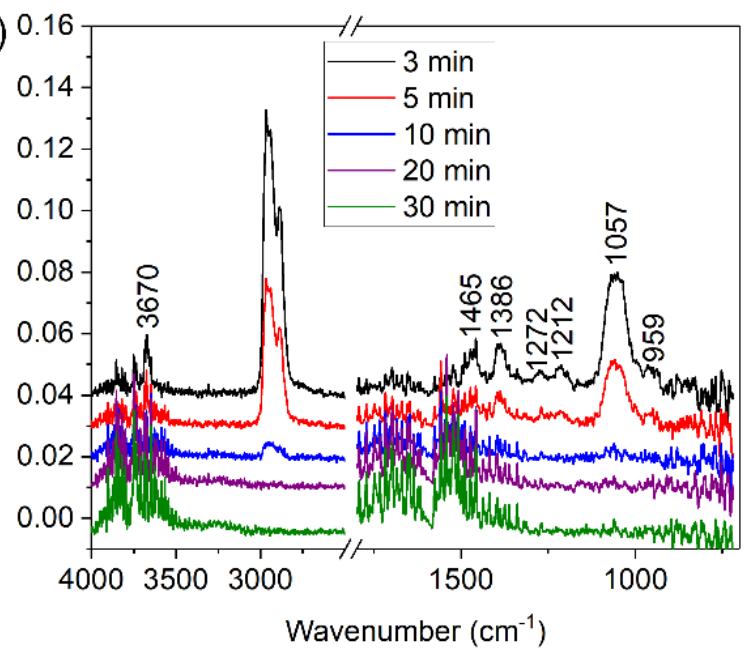

(c)

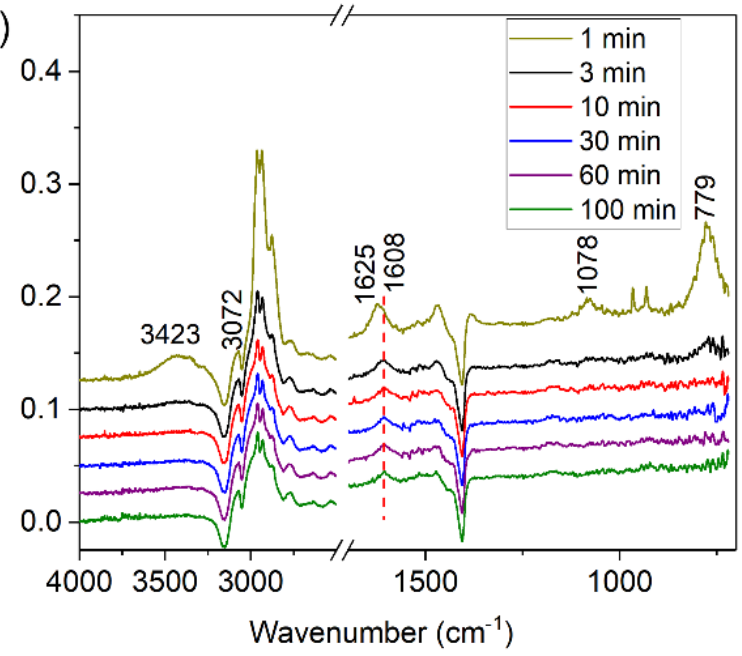

(b)

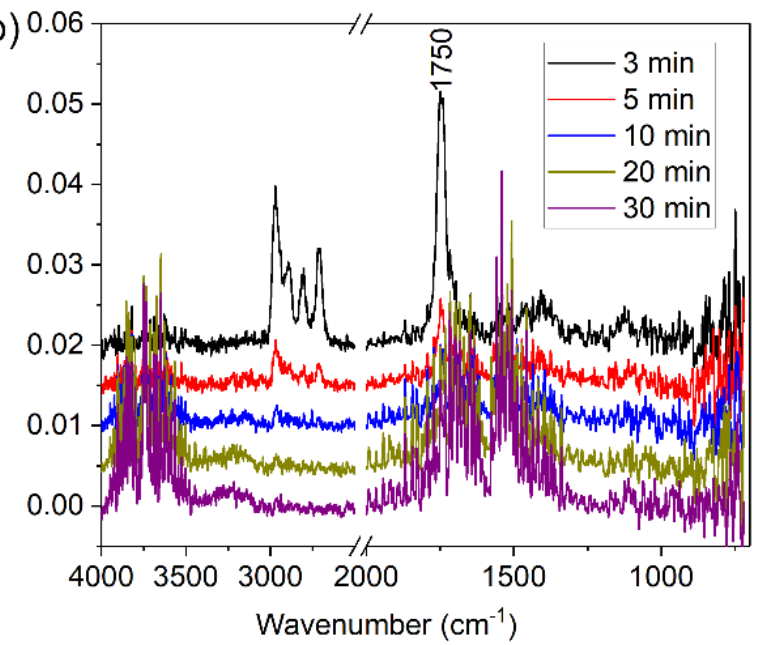

(d)

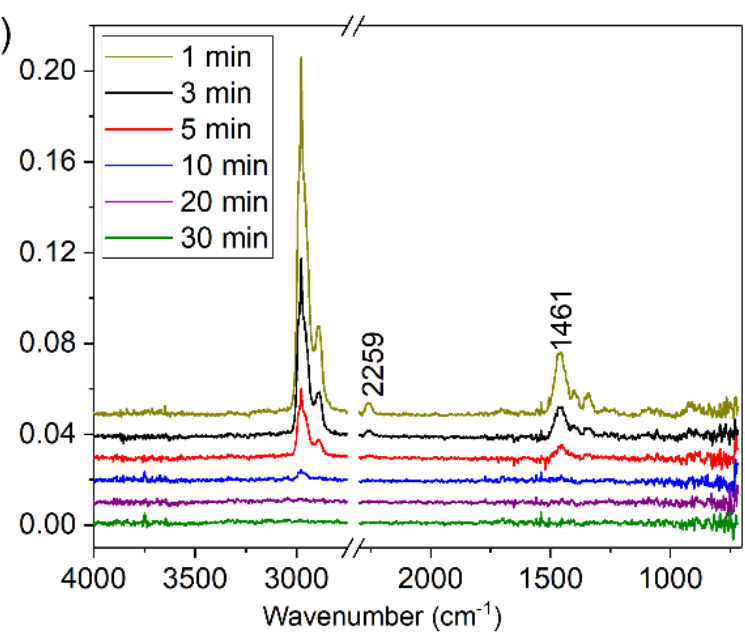

Figure S6. DRIFTS of desorption behaviour of (a) 1-butanol, (b) butanal, (c) 1-butylamine and (d) butyronitrile on $\mathrm{NiCu}$ surface. At $\mathrm{t}=0,10 \mu \mathrm{l}$ of organic compounds were injected under $\mathrm{N}_{2}$ flow, $130{ }^{\circ} \mathrm{C}$. 
(a)

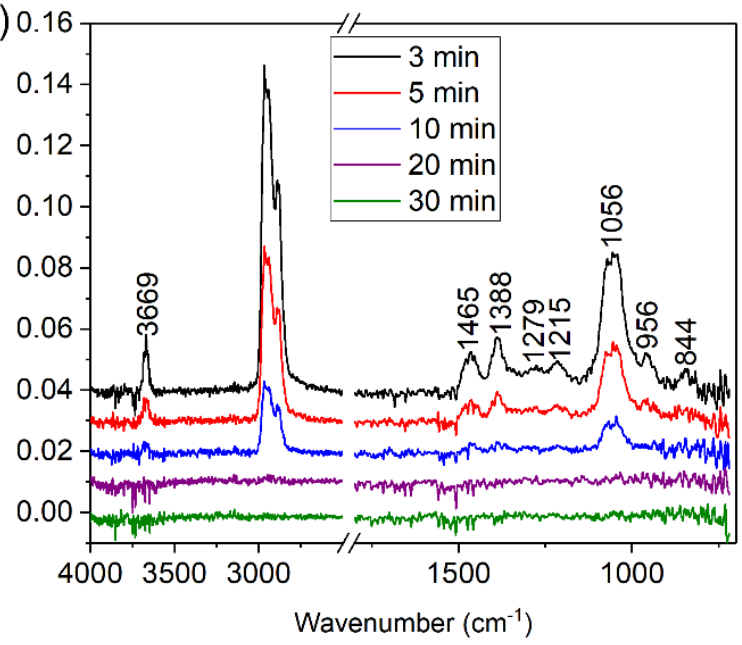

(c) 0.8

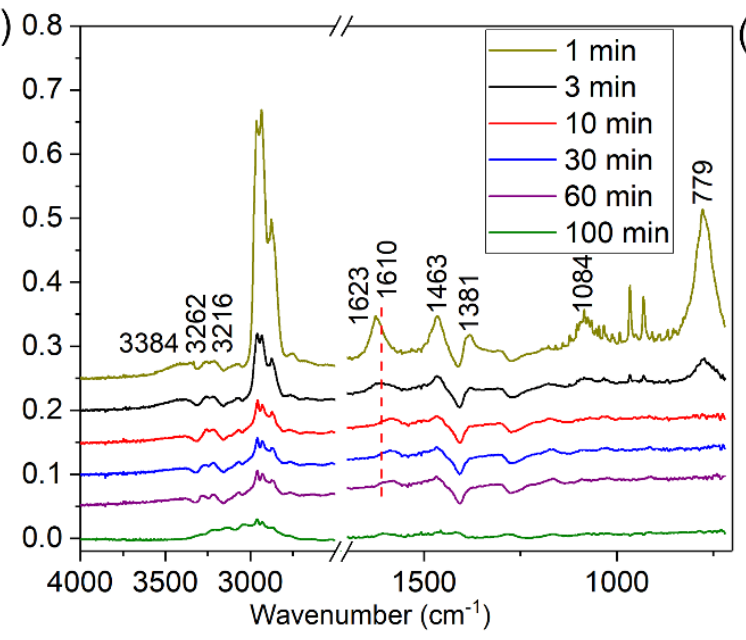

(b)

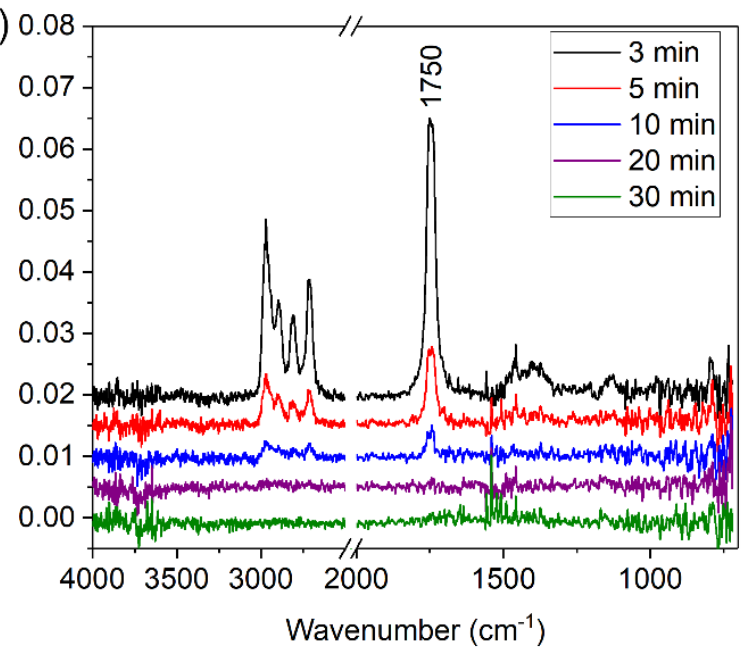

(d)

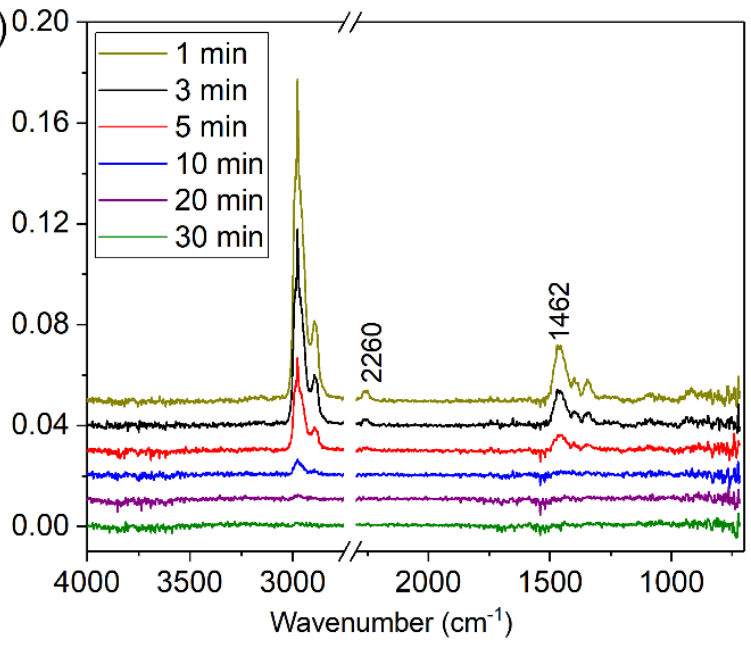

Figure S7. DRIFTS of desorption behaviour of (a) 1-butanol, (b) butanal, (c) 1-butylamine and (d) butyronitrile on Ni surface. At $\mathrm{t}=0,10 \mu \mathrm{l}$ of organic compounds were injected under $\mathrm{N}_{2}$ flow, $130{ }^{\circ} \mathrm{C}$. 
(a) 0.16

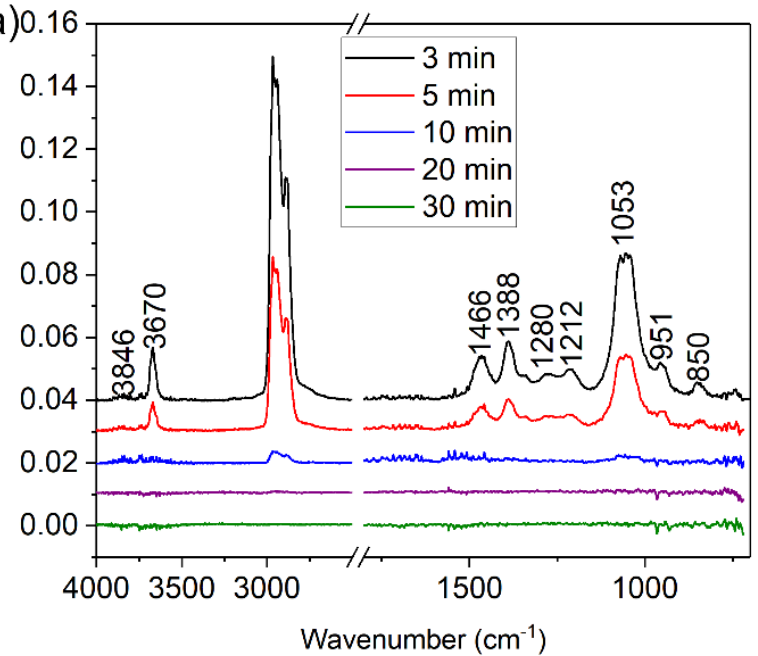

(c)

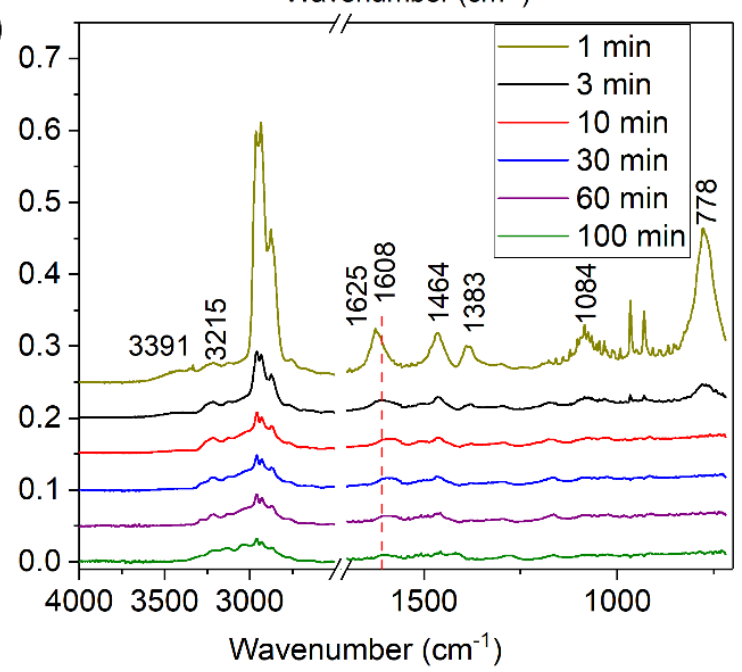

(b) 0.07

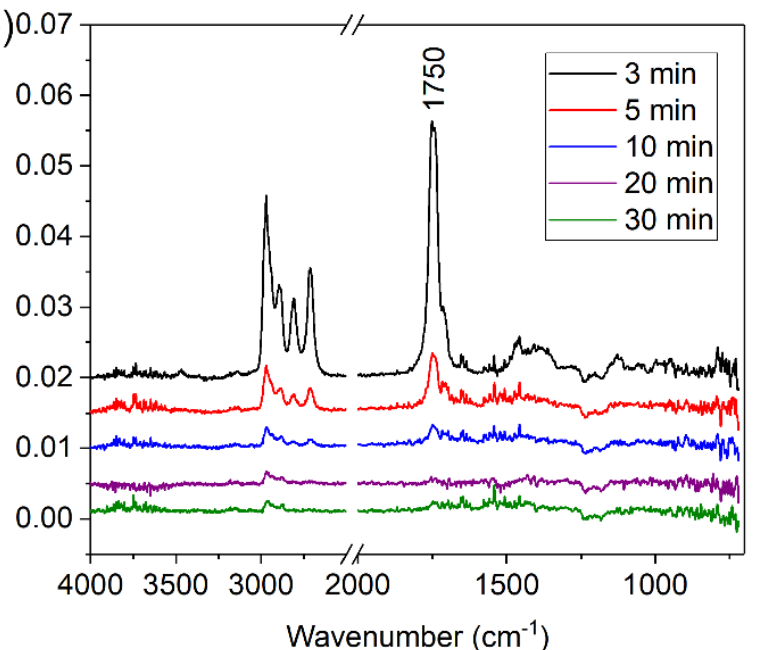

(d) 0

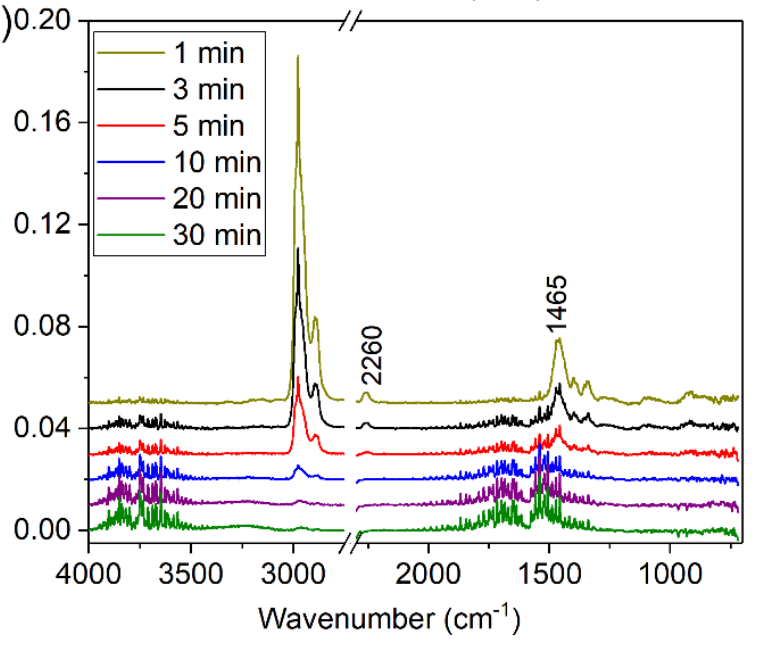

Figure S8. DRIFTS of desorption behaviour of (a) 1-butanol, (b) butanal, (c) 1-butylamine and (d) butyronitrile on $\mathrm{Cu}$ surface. At $\mathrm{t}=0,10 \mu \mathrm{l}$ of organic compounds were injected under $\mathrm{N}_{2}$ flow, $130{ }^{\circ} \mathrm{C}$. 


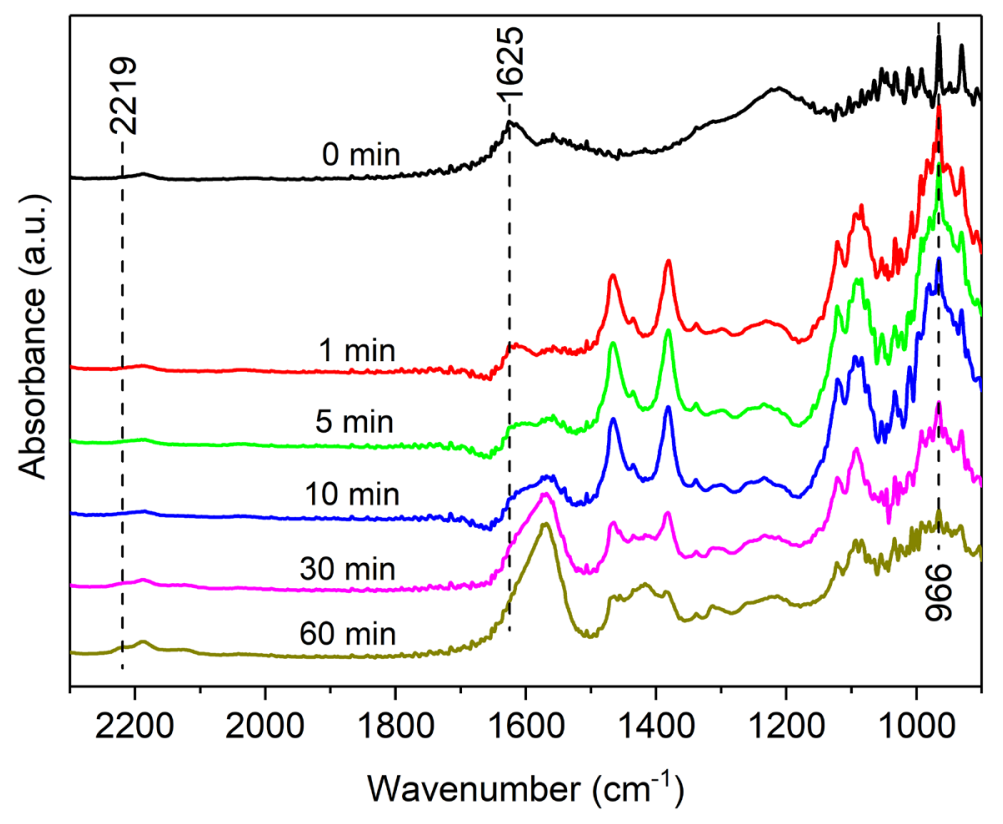

Figure S9. DRIFTS of desorption behaviour of 1-butanol on $\mathrm{NH}_{3}$ pre-adsorbed $\mathrm{Ni}_{0.5} \mathrm{Cu}_{0.5} / \mathrm{Al}_{2} \mathrm{O}_{3}$ surface. At $\mathrm{t}=0,10 \mu \mathrm{l}$ of 1-butanol was injected under $\mathrm{N}_{2}$ flow, $130{ }^{\circ} \mathrm{C}$. 


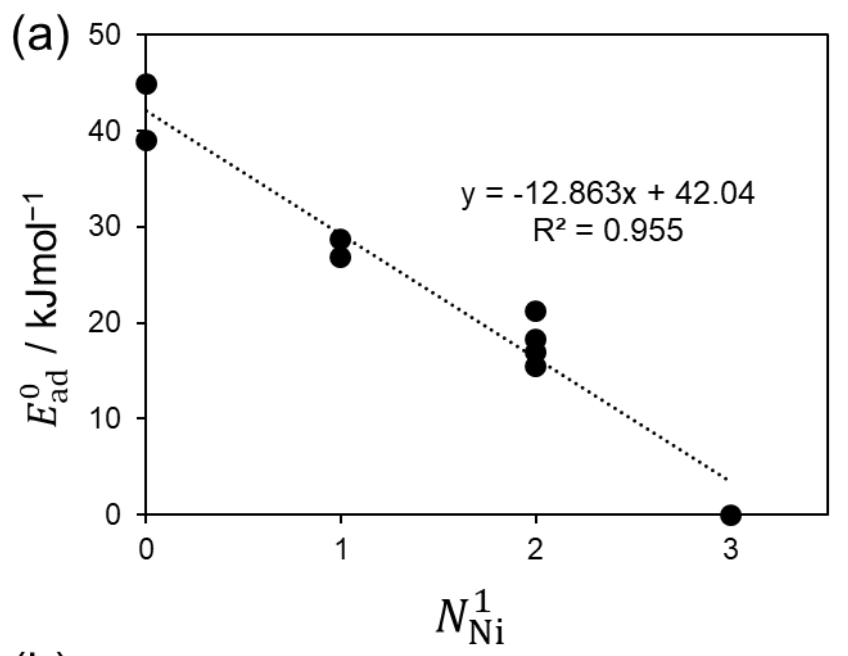

(c)

(d)
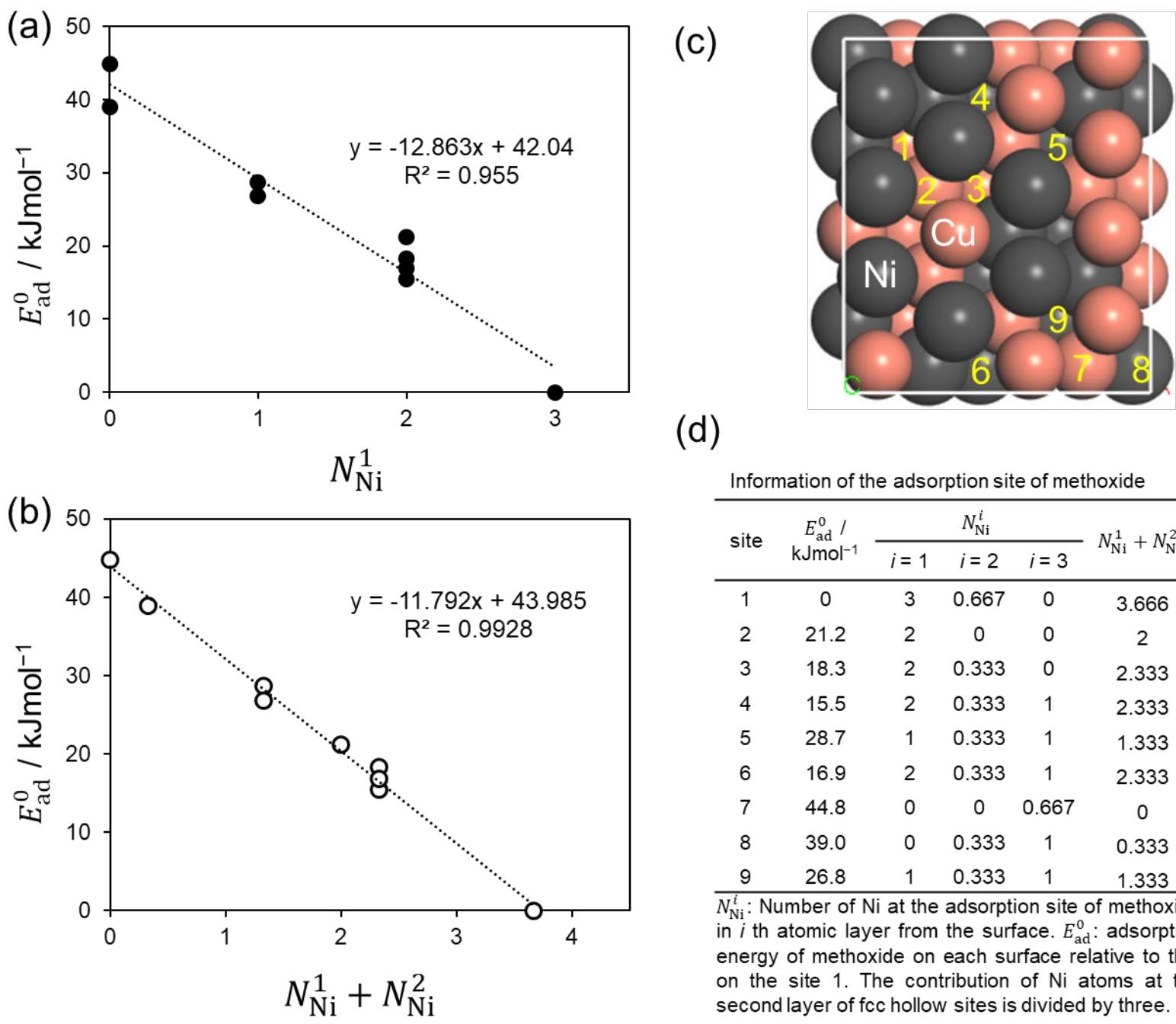

\begin{tabular}{|c|c|c|c|c|c|}
\hline \multirow{2}{*}{ site } & \multirow{2}{*}{$\begin{array}{c}E_{\mathrm{ad}}^{0} / \\
\mathrm{kJmol}^{-1}\end{array}$} & \multicolumn{3}{|c|}{$N_{\mathrm{Ni}}^{i}$} & \multirow{2}{*}{$N_{\mathrm{Ni}}^{1}+N_{\mathrm{Ni}}^{2}$} \\
\hline & & $i=1$ & $i=2$ & $i=3$ & \\
\hline 1 & 0 & 3 & 0.667 & 0 & 3.666 \\
\hline 2 & 21.2 & 2 & 0 & 0 & 2 \\
\hline 3 & 18.3 & 2 & 0.333 & 0 & 2.333 \\
\hline 4 & 15.5 & 2 & 0.333 & 1 & 2.333 \\
\hline 5 & 28.7 & 1 & 0.333 & 1 & 1.333 \\
\hline 6 & 16.9 & 2 & 0.333 & 1 & 2.333 \\
\hline 7 & 44.8 & 0 & 0 & 0.667 & 0 \\
\hline 8 & 39.0 & 0 & 0.333 & 1 & 0.333 \\
\hline 9 & 26.8 & 1 & 0.333 & 1 & 1.333 \\
\hline \multicolumn{6}{|c|}{$\begin{array}{l}N_{\mathrm{Ni}}^{i}: \text { Number of } \mathrm{Ni} \text { at the adsorption site of methoxide } \\
\text { in } i \text { th atomic layer from the surface. } E_{\mathrm{ad}}^{0}: \text { adsorption } \\
\text { energy of methoxide on each surface relative to tha } \\
\text { on the site } 1 \text {. The contribution of } \mathrm{Ni} \text { atoms at the } \\
\text { second layer of fcc hollow sites is divided by three. }\end{array}$} \\
\hline
\end{tabular}

Figure S10. Correlation between Ead of methoxide and the number of $\mathrm{Ni}$ atoms at the adsorption site without (a) and (b) with correction considering the contribution of the Ni atoms in the second layer. (c) Structure of adsorption hollow sites considered in (a) and (b). (d) summary and details of the adsorption site of methoxide. 
(a)

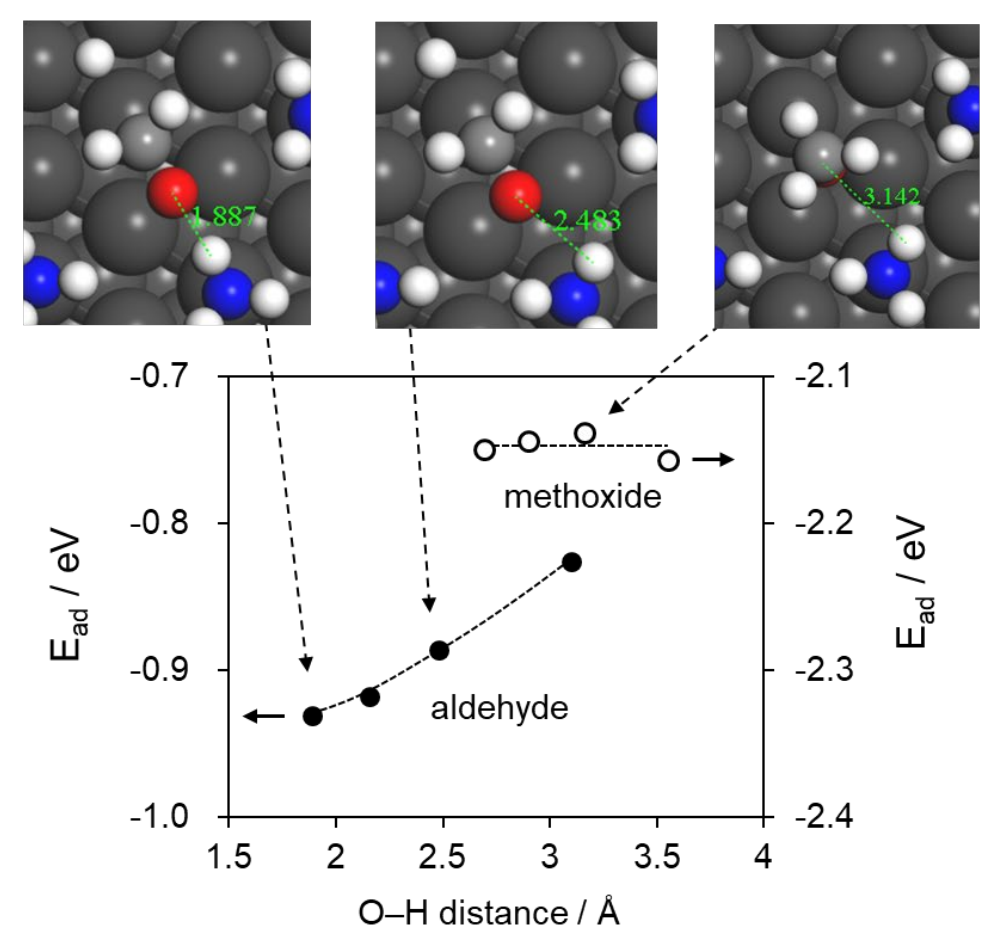

(b)

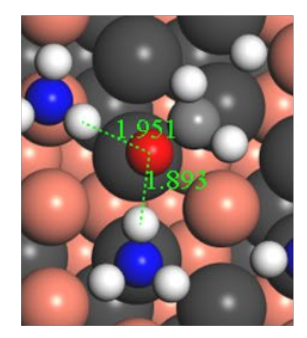

$\mathrm{NiCu}-2+$ $3 \mathrm{NH}_{3}(2 \mathrm{HB})$

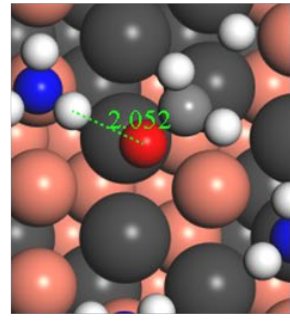

$\mathrm{NiCu}-2+$ $3 \mathrm{NH}_{3}(1 \mathrm{HB})$

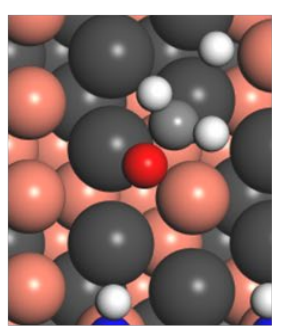

$\mathrm{NiCu}-2+$ $2 \mathrm{NH}_{3}(\mathrm{OHB})$

Figure S11. (a) Correlation between $\mathrm{E}_{\mathrm{ad}}$ (methoxide or formaldehyde) and $\mathrm{O}-\mathrm{H}$ distance regarding hydrogen bonding (HB). (b) Structures of formaldehyde and ammonia with various fashions of hydrogen bonding. 


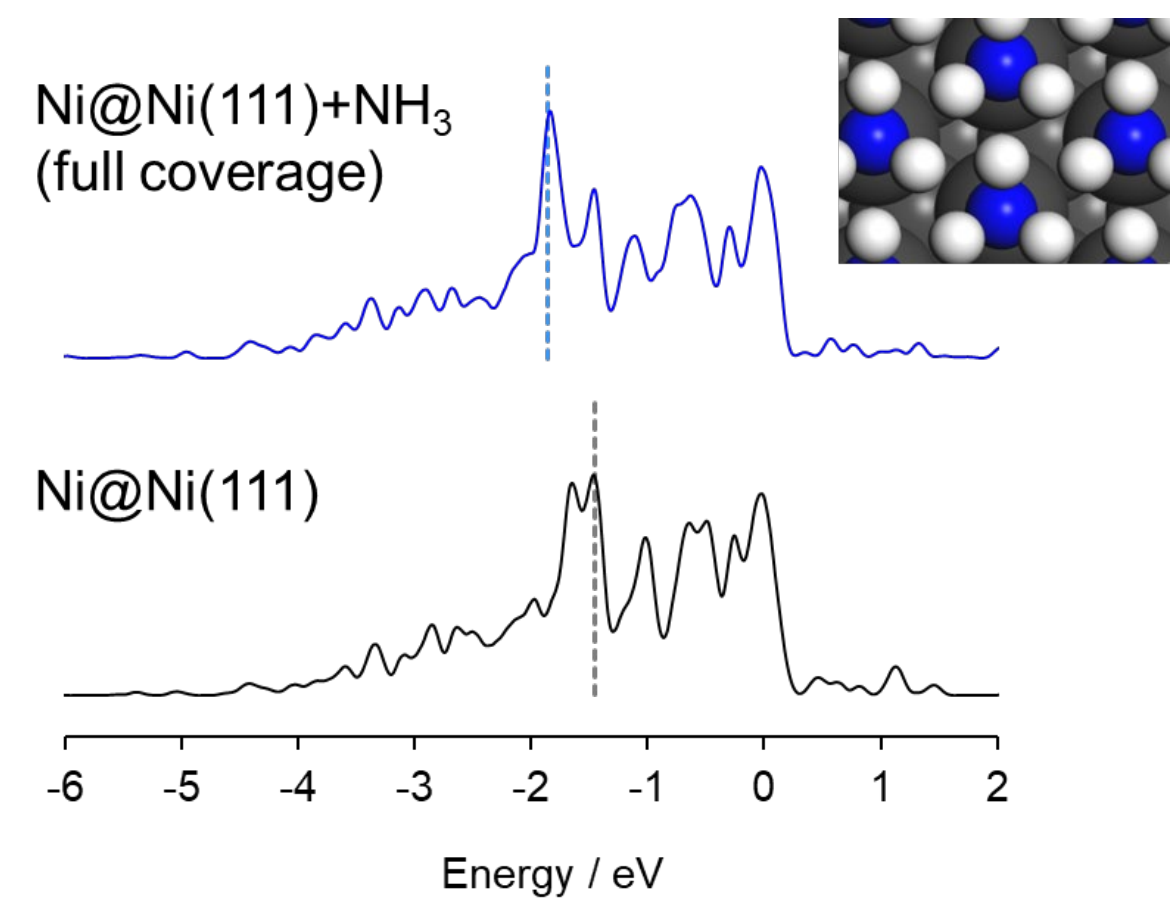

Figure S12. Density of state diagrams projected on d orbitals of surface Ni atoms of Ni(111) with and without ammonia. Vertical dotted lines indicate the position of $\mathrm{d}$ band center. 
$\odot \mathrm{Ni} \odot \mathrm{Cu} \odot \mathrm{Zn} \odot \mathrm{C} \odot \mathrm{O} \odot \mathrm{N} \odot \mathrm{H} \odot$ methoxide formaldehyde $+\mathrm{H}$

(a)

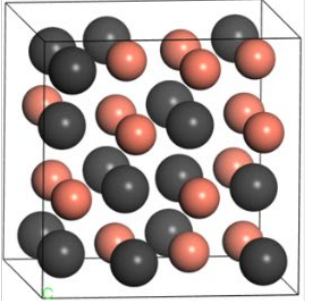

$\mathrm{Ni}_{0.5} \mathrm{M}_{0.5}(\mathrm{M}=\mathrm{Fe}, \mathrm{Co}, \mathrm{Cu})$

(b)

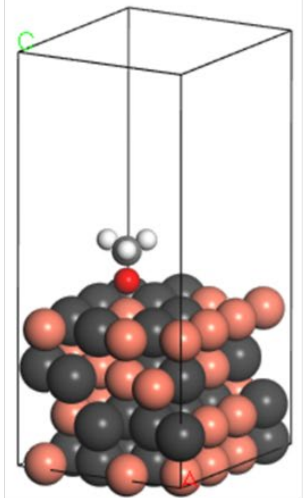

methoxide on $\mathrm{Ni}_{0.5} \mathrm{Cu}_{0.5}(111)$

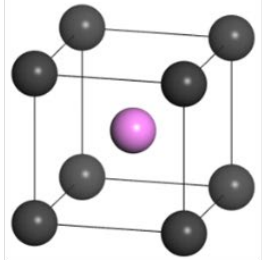

$\mathrm{NiZn}(\mathrm{P} 4 / \mathrm{mmm})$
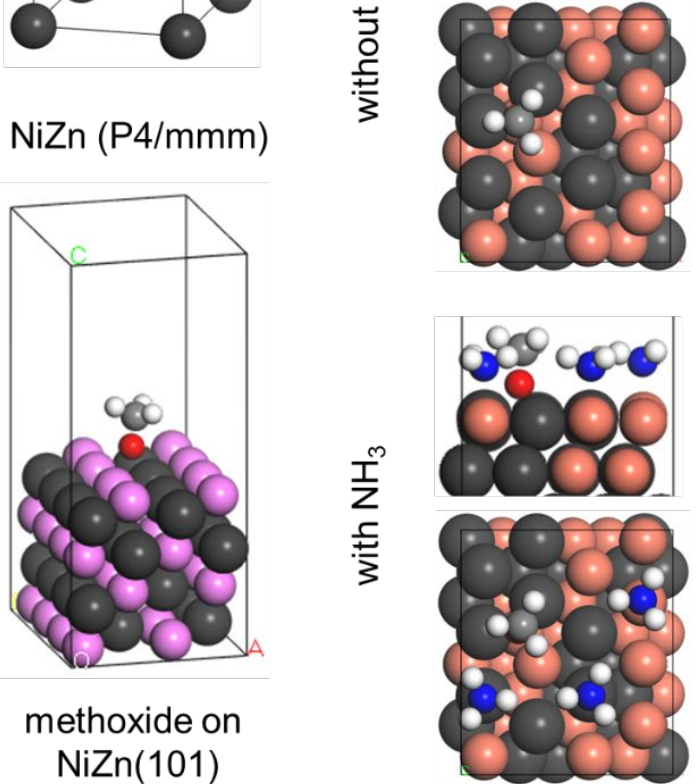

(c)
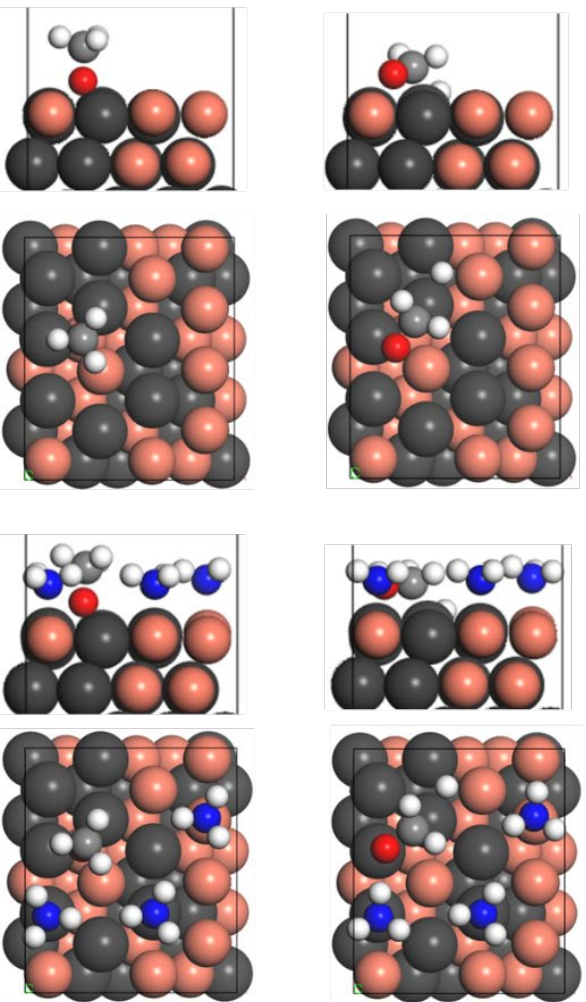

Figure S13. (a) Structure model of $\mathrm{Ni}_{0.5} \mathrm{M}_{0.5}$ solid-solution alloys and NiZn intermetallic compound. (b) Slab models with methoxide adsorbed on $\mathrm{Ni}_{0.5} \mathrm{M}_{0.5}(111)$ and $\mathrm{NiZn}(101)$ surfaces. (c) optimized structures of adsorbates on $\mathrm{Ni}_{0.5} \mathrm{Cu}_{0.5}(111)$ surface. For each surface, a $\mathrm{Ni}_{2} \mathrm{M}$ hollow was chosen as the adsorption site for methoxide, formaldehyde, and $\mathrm{H}$. 


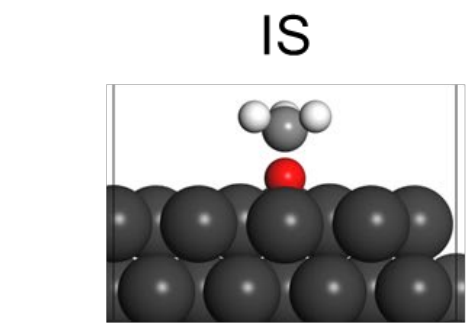

$\bar{z}$
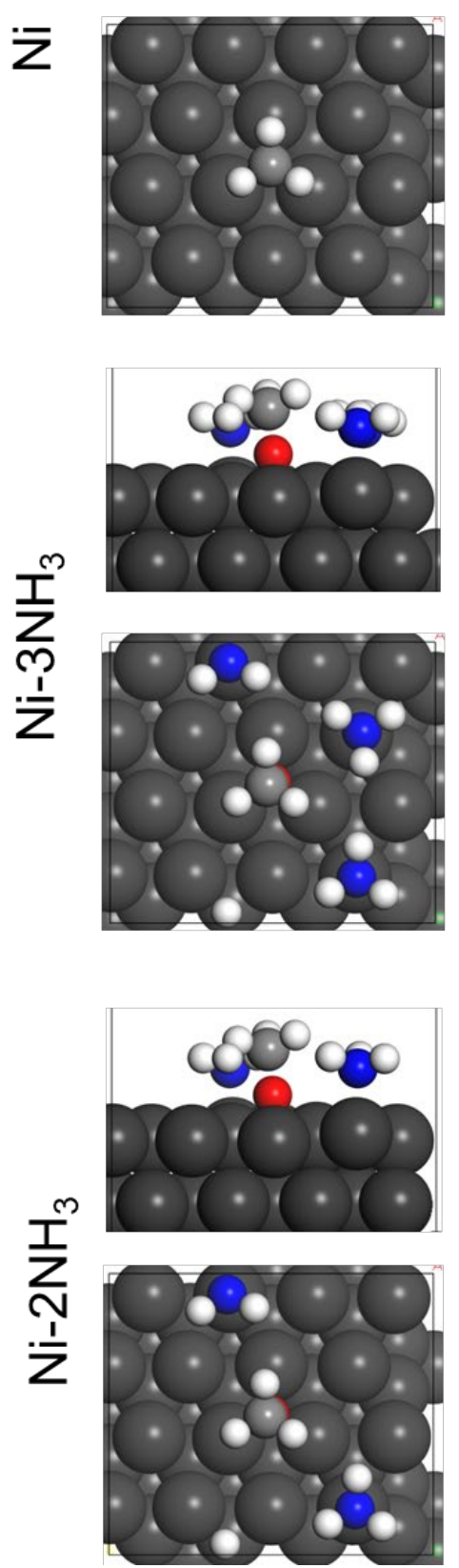

TS

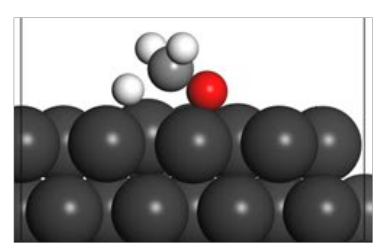

FS
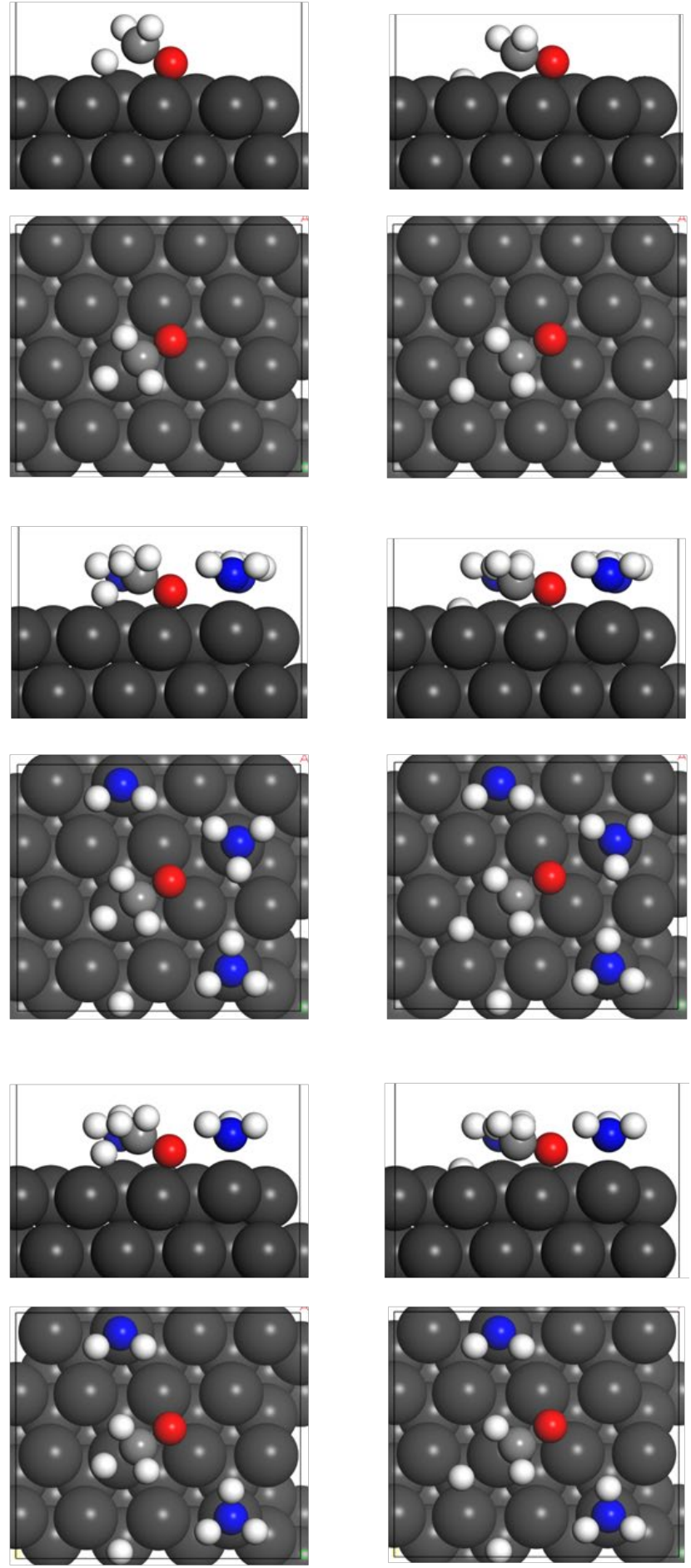

Figure S14. Pictures of initial (IS), transition (TS), and final states (FS) for C-H activation of methoxide adsorbed on $\mathrm{Ni}(111)$ with and without ammonia co-adsorbed. 


\section{$\mathrm{NiCu}-1$}
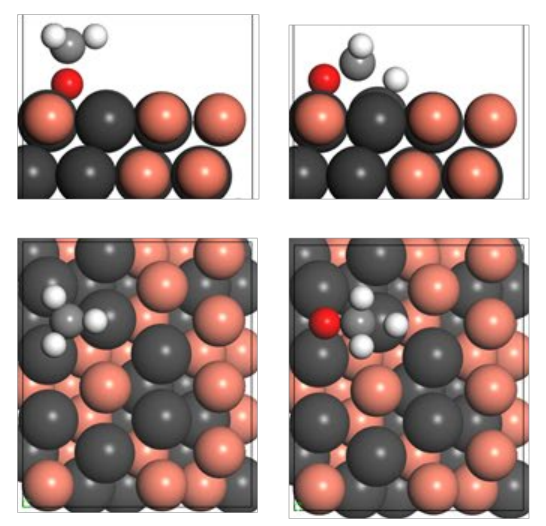

$\mathrm{NiCu}-3$
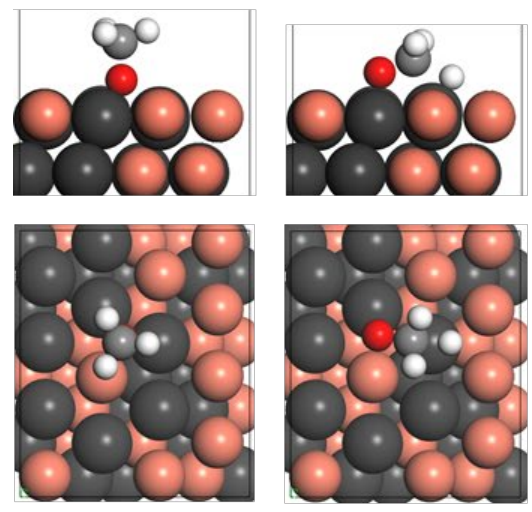

$\mathrm{NiCu}-5$
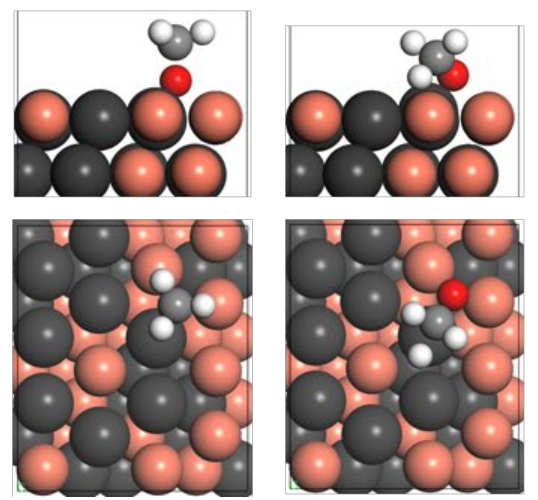
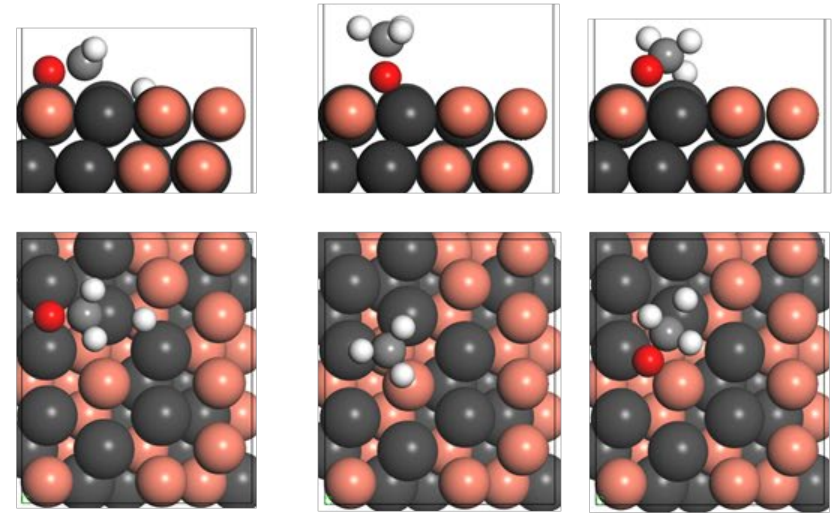

$\mathrm{NiCu}-4$
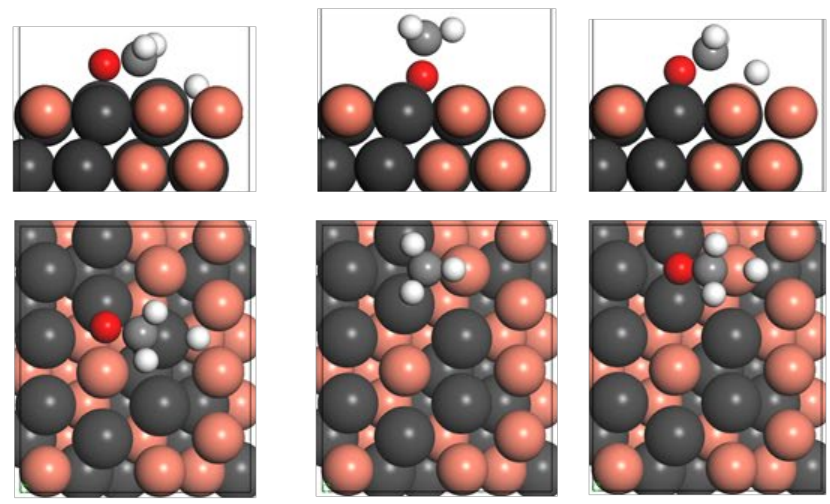

NiCu-6
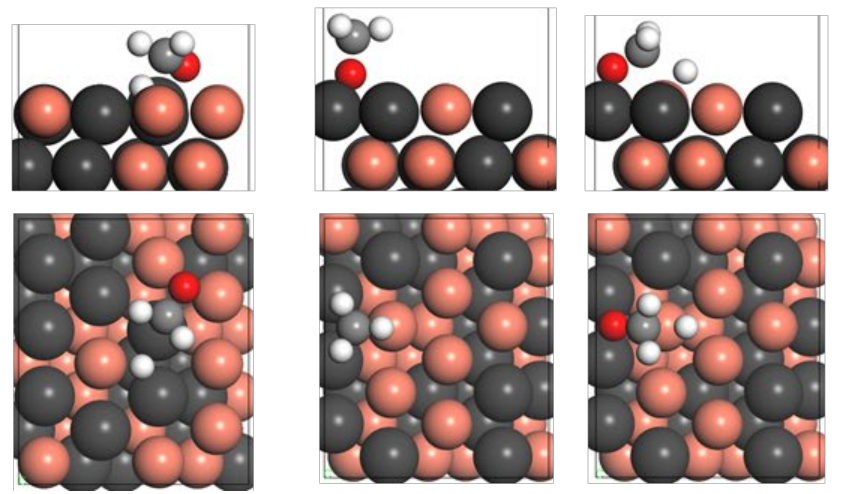
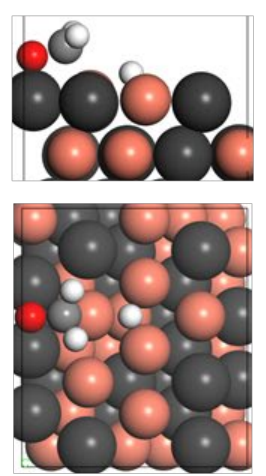

Figure S15. Pictures of IS, TS, and FS for $\mathrm{C}-\mathrm{H}$ activation of methoxide adsorbed on $\mathrm{NiCu}(111)$ surface with various adsorption configurations. 
$\mathrm{NiCu}-2(2 \mathrm{HB})$
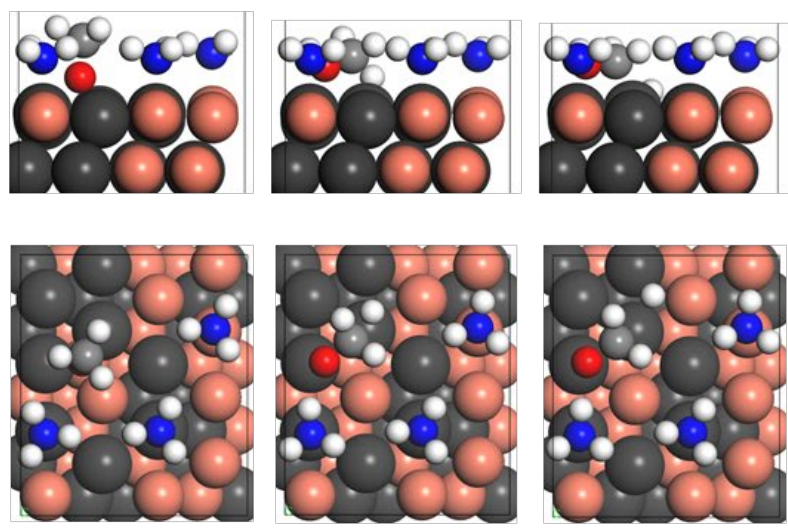

$\mathrm{NiCu}-2(\mathrm{OHB})$
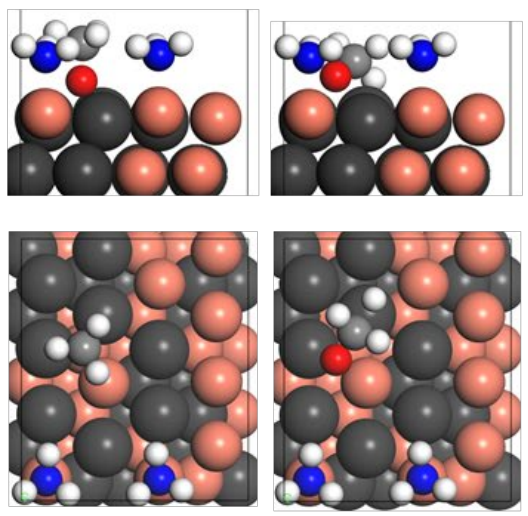

$\mathrm{NiCu}-5$ (2HB)
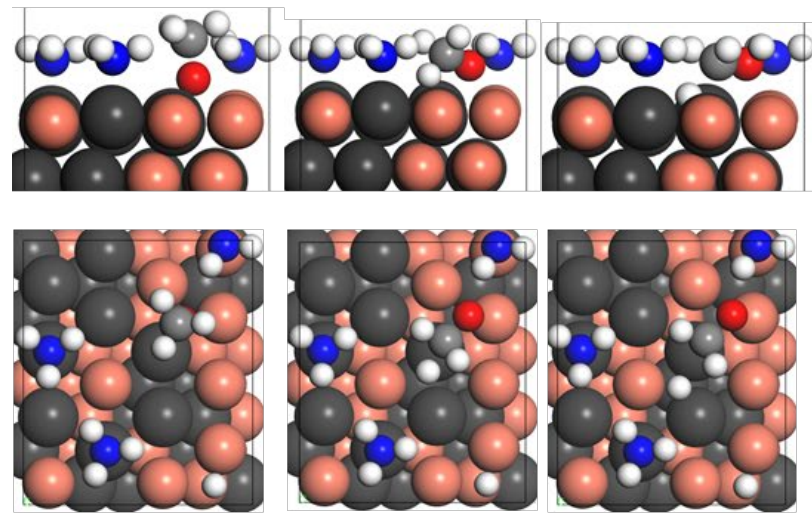

$\mathrm{NiCu}-2(1 \mathrm{HB})$
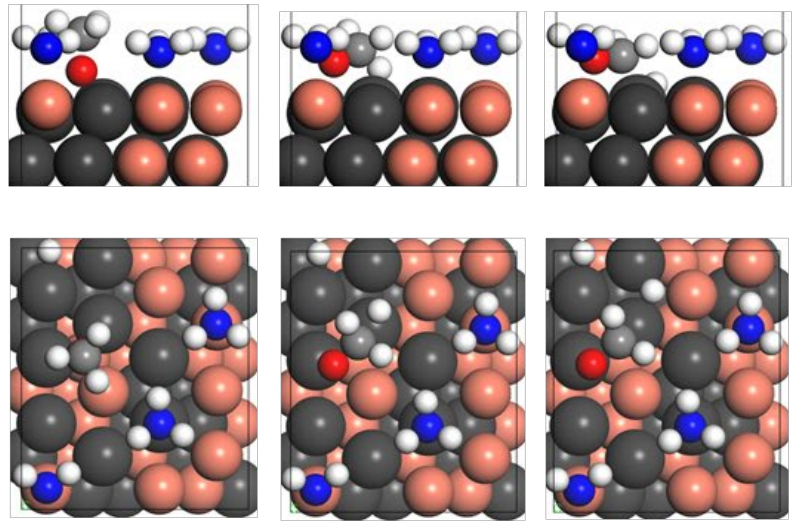

$\mathrm{NiCu}-4(2 \mathrm{HB})$
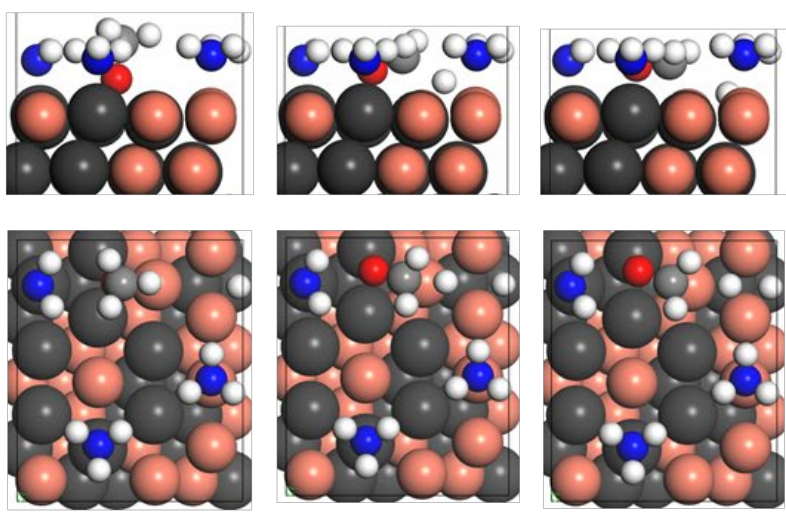

$\mathrm{NiCu}-6(2 \mathrm{HB})$
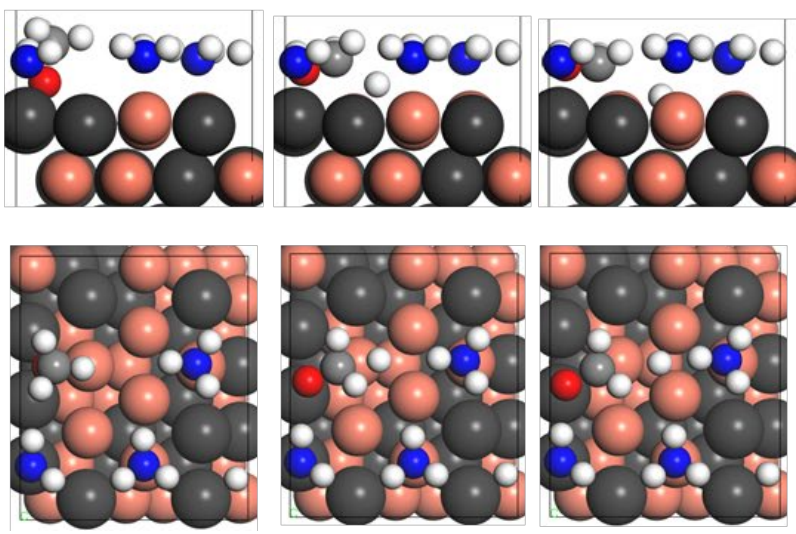

Figure S16. Pictures of IS, TS, and $\mathrm{FS}$ for $\mathrm{C}-\mathrm{H}$ activation of methoxide adsorbed on $\mathrm{NiCu}(111)$ surface with ammonia co-adsorbed and with various adsorption configurations . 
NiFe-2
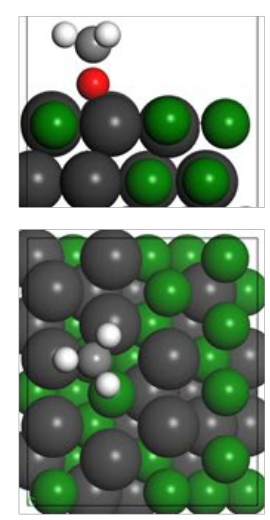
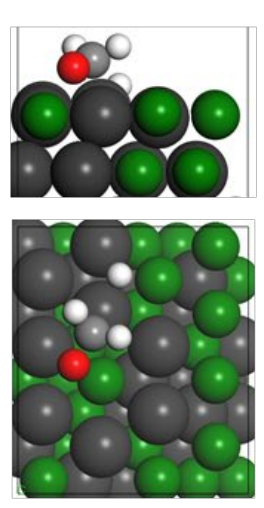

NiCo-2
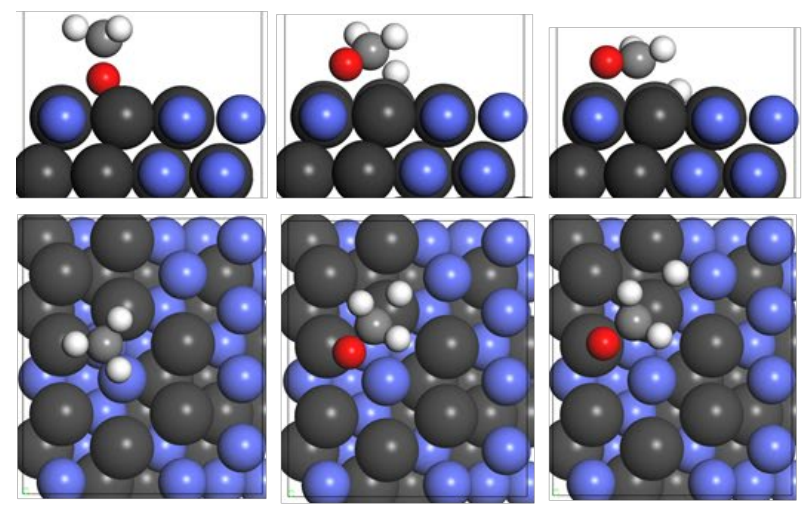
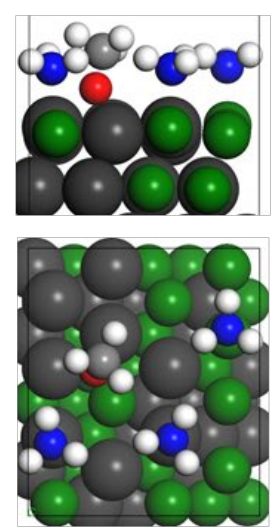

NiFe-2 (2HB)
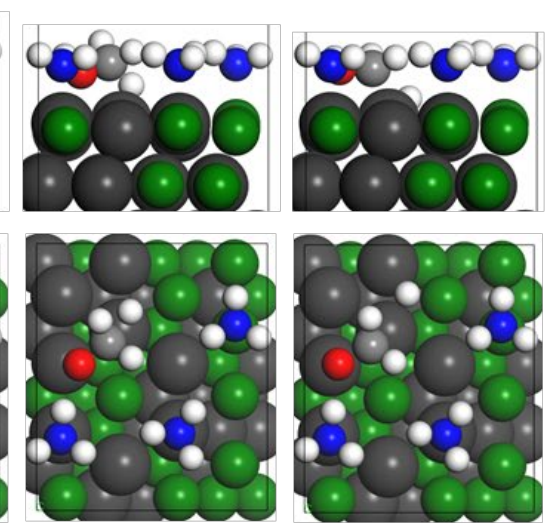

NiCo-2 (2HB)
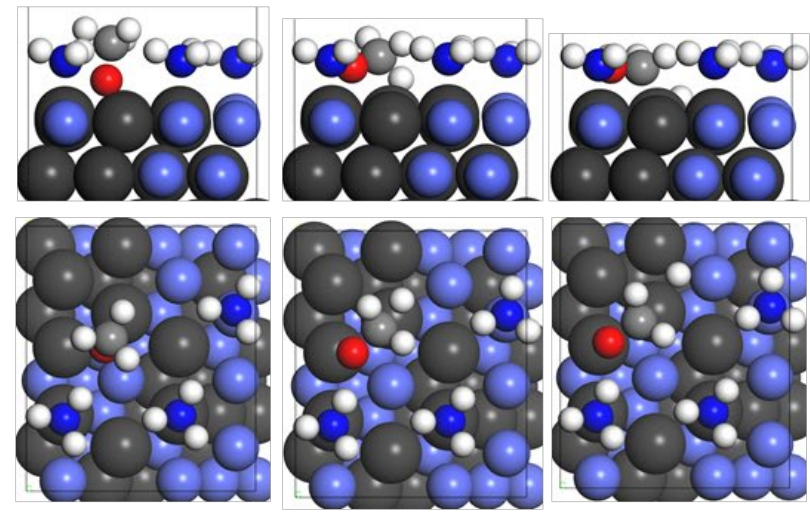

$\mathrm{NiZn}(2 \mathrm{HB})$
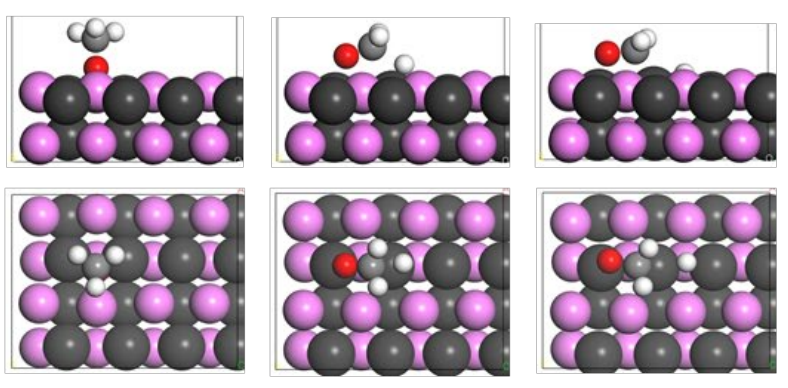

Figure S17. Pictures of IS, TS, and FS for $\mathrm{C}-\mathrm{H}$ activation of methoxide adsorbed on $\mathrm{NiFe}(111)$, $\mathrm{NiCo}(111)$ and NiZn(101) surface with and without ammonia co-adsorbed. 


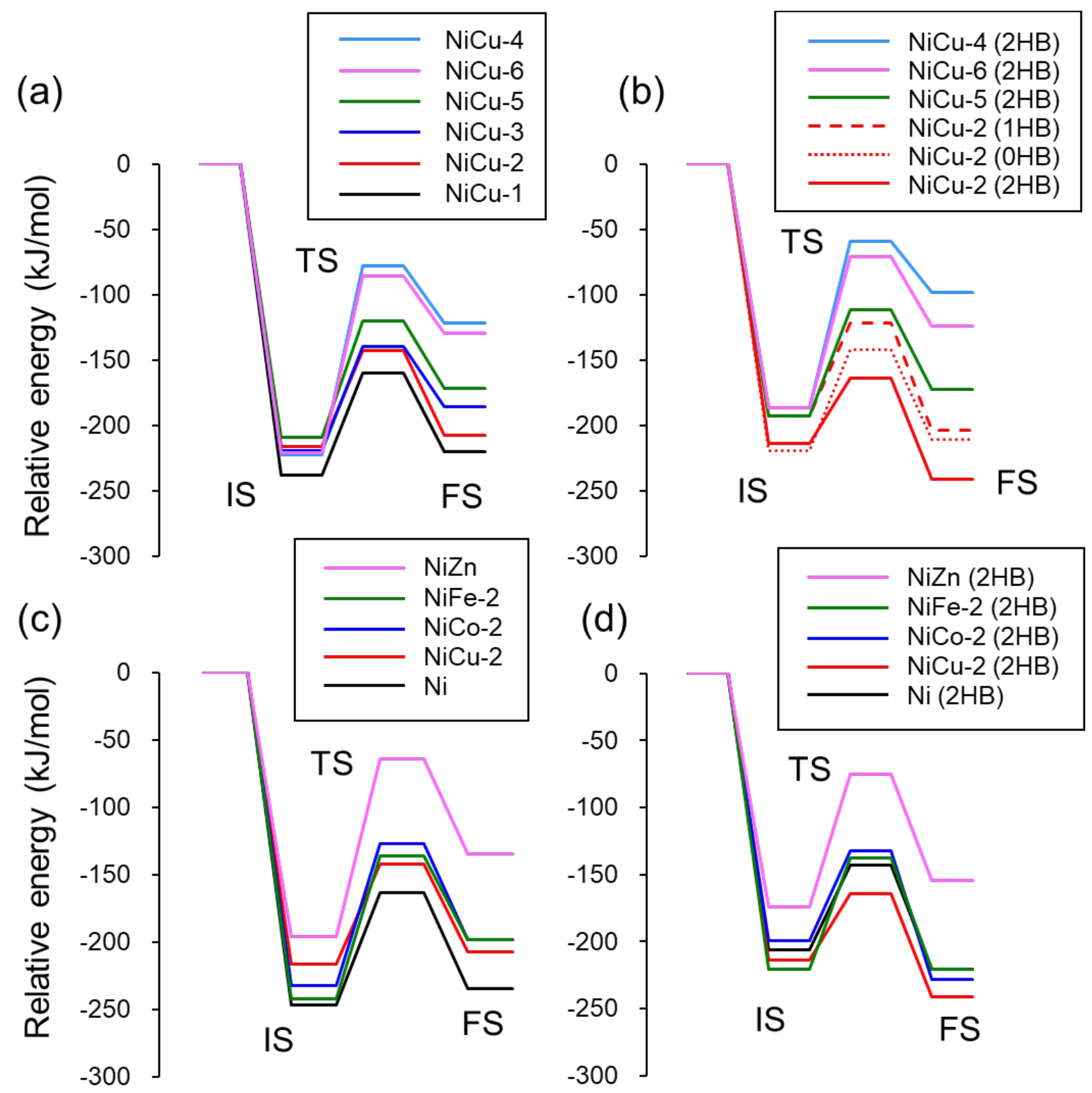

Figure S18. Energy diagrams for $\mathrm{C}-\mathrm{H}$ activation of methoxide to formaldehyde on various sites of $\mathrm{NiCu}$ (a) without and (b) with ammonia and $\mathrm{Ni}$ and NiM surfaces (c) without and (d) with ammonia. IS (initial state): adsorbed methoxide, TS (transition state), and FS (final state): adsorbed formaldehyde and hydrogen atom. The corresponding energy values are also shown in Table S7. Notation in parenthesis indicates the number of hydrogen bonding (HB). Total energy of the slab (with or without co-adsorbed ammonia) and free methoxide was set to zero. 


\section{References}

(1) Ravel, B.; Newville, M. ATHENA, ARTEMIS, HEPHAESTUS: Data Analysis for X-ray Absorption Spectroscopy using IFEFFIT. J. Synchrotron Rad. 2005, 12, 537-541.

(2) Miyazaki, M.; Furukawa, S.; Komatsu, T. Regio- and Chemoselective Hydrogenation of Dienes to Monoenes Governed by a Well-Structured Bimetallic Surface. J. Am. Chem. Soc. 2017, 139, 18231-18239.

(3) Tkatchenko, A.; Scheffler, M. Accurate Molecular Van Der Waals Interactions from Ground-State Electron Density and Free-Atom Reference Data. Phys. Rev. Lett. 2009, 102, 073005.

(4) Monkhorst, H. J.; Pack, J. D. Special Points for Brillouin-Zone Integrations. Phys. Rev. B 1976, 13, 5188-5192.

(5) Halgren, T. A.; Lipscomb, W. N. Synchronous-Transit Method for Determining Reaction Pathways and Locating Molecular Transition-States. Chem Phys Lett 1977, 49, 225-232.

(6) Govind, N.; Petersen, M.; Fitzgerald, G.; King-Smith, D.; Andzelm, J. A Generalized Synchronous Transit Method for Transition State Location. Comput. Mater. Sci. 2003, 28, 250258. 